\title{
Numerical Solution of the Nonlocal Hammerstein-Volterra Integral Equation with Continuous Kernels
}

\author{
M.M.El-Kojok \\ Department of Mathematics, College of Sciences and Arts, Qassim University, K.S.A.
}

\begin{abstract}
In this paper, after using Picard's method, the existence and uniqueness solution of the nonlocal Hammerstein-Volterra integral equation $(\boldsymbol{H}-\boldsymbol{V I E})$ of the second kind with continuous kernels are considered. Using two different numerical methods Trapezoidal rule and Simpson's rule, the nonlocal H-VIE, in each method, is conformal to a nonlocal nonlinear algebraic system (nonlocal NAS). Some numerical results are calculated, when the nonlocal term is neglected, in the linear case and in the nonlinear case. Moreover, the error estimate, in each case, is computed. Many special cases are derived from the work when the memory takes different cases. In addition, the HI termand VI term take the linear and nonlinear cases.
\end{abstract}

Keywords: Nonlocal Hammerstein-Volterra integral equation, Picard's method,Trapezoidal rule, Simpson's rule, nonlocal nonlinear algebraic system.

MSC(2010): 45B05;45G10;65R .

\section{Introduction}

Many problems of mathematical physics, contact problems in the theory of elasticity and mixed problems of mechanics of continuous media reduce to an integral equation with continuous or discontinuous kernels. The integral equations have received considerable interest of many applications in different mathematical areas of sciences, see [1-8]. Therefore, different analytic and numeric methods have been established to obtain the solutions of the integral equations. For analytical methods, we state degenerate kernel method, Cauchy method (singular integral method), Laplace transformation method, Fourier transformation method, potential theory method, and Krien's method, see [9-12].More informations for the analytic methods can be found in Muskhelishvili [13], Popov [14], Tricomi [15], Hochstad [16] and Green [17]. More recently, since analytical methods on practical problems often fail, numerical solutions of these equations are a much studied subjected of numerous works. The interested reader should consult the fine exposition by Atkinson [18], Delves and Mohamed [19], Golberg [20] and Linz [21] for some different numerical methods.

In most previous works of the authors, in solving linear and nonlinear integral equations, the historical memory of the problem is neglected. For this, in this work the effect of the historical memory in the numerical results in the nonlinear integral equations will discussed.

Consider, in the space $C[0, T], T<1$, the nonlocal H-VIE of the second kind:

$$
\mu \varphi(t)=f(t, H(t, \varphi(t)))+\lambda_{1} \int_{0}^{1} k(t, s) \gamma(s, \varphi(s)) d s+\lambda_{2} \int_{0}^{t} F(t, s) g(s, \varphi(s)) d s .
$$

Here, the given functions $f(t, H(t, \varphi(t)))$ and $H(t, \varphi(t))$ are called the free term and the memory of the integral equation (1.1), respectively.The two functions $\gamma(t, \varphi(t))$ and $g(t, \varphi(t))$ are given, while the function $\varphi(t)$ is unkown in the space $C[0, T], T<1$. The two known functionsk $(t, s)$ and $F(t, s)$ are continuous and called thekernels of HIterm andVIterm, respectively. The constant $\mu$ defines the kind of the integral equation,and each of the constants $\lambda_{1}$ and $\lambda_{2}$ has a physical meaning.

The proposeof this paper, isproving the existence of a unique solution of the nonlocal H-VIE (1.1)under certain conditionsusing the method of successive approximations (Picard's method). In addition, weuse two different numerical methods Trapezoidal rule, and Simpson's rule for reducing the nonlocal H-VIE to a nonlocal nonlinear algebraic systemof equations(nonlocal NAS) which will be solved numerically.Finally, numerical results are calculated and the error estimate, in each method, is computed.

\section{The Existence and Uniqueness Solution of ThenonlocalH-VIE}

In this section,the successive approximations (Picard's method) will be considered to prove the existence of a unique solution $\varphi(t)$ of Eq. (1.1), in the Banach space $C[0, T], T<1$, under the following conditions:

(i) The given functions $f(t, H(t, \varphi(t))), \gamma(t, \varphi(t))$ and $g(t, \varphi(t))$ with their derivatives for all $t \in$ $[0, T]$ belong to $C[0, T]$, andsatisfy for the constants $p_{1}, p_{2}, p_{3}$ and $p \geq \max \left\{p_{\ell}\right\} ; \ell=1,2,3$, the following conditions: 
(a) $|f(t, H(t, \varphi(t)))| \leq p_{1}|\varphi(t)|,(b)|\gamma(t, \varphi(t))| \leq p_{2}|\varphi(t)|$,

(c) $|g(t, \varphi(t))| \leq p_{3}|\varphi(t)|$.

Moreover, for the function $L(t)>\max _{0 \leq t \leq T}\left\{L_{\ell}(t)\right\} ; \ell=1,2,3$, and for theconstants $\mathcal{L}>\max _{0 \leq t \leq T}|L(t)|$ and $q \geq \max \{p, \mathcal{L}\}$, we have

(d) $|f(t, H(t, \varphi(t)))-f(t, H(t, \psi(t)))| \leq L_{1}(t)|\varphi(t)-\psi(t)|$,

(e) $|\gamma(t, \varphi(t))-\gamma(t, \psi(t))| \leq L_{2}(t)|\varphi(t)-\psi(t)|$,

(f) $|g(t, \varphi(t))-g(t, \psi(t))| \leq L_{3}(t)|\varphi(t)-\psi(t)|$.

(ii) The continuous kernelsk $(t, s)$ and $F(t, s)$, for all $t, s \in[0, \mathrm{~T}]$, satisfy $|k(t, s)| \leq M,|F(t, s)| \leq$ $S,(M, S$ are constants).

(iii) Thenorm of thefunction $f(t, 0)=f(t)$ is defined by : $\|f(t)\|=\max _{0 \leq t \leq T}|f(t)| \leq G,(G$ is constant).

Theorem 1: The nonlocal H-VIE (1.1) has a unique solution in the Banach $\operatorname{space} C[0, T]$ under the condition : $q\left(1+\left|\lambda_{1}\right| M+\left|\lambda_{2}\right| T S\right)<|\mu|$.

The proof of this theorem depends on the following lemmas.

Lemma 1: Under the conditions (i)-(iii), the infinite $\operatorname{series} \sum_{s=0}^{\infty} V_{s}(t)$ is uniformly convergent to a continuous function $\varphi(t)$.

Proof:Construct a sequence of functions $\left\{\varphi_{n}(t)\right\}$, as follow

$$
\begin{aligned}
\mu \varphi_{n}(t)=f\left(t, H\left(t, \varphi_{n-1}(t)\right)\right)+\lambda_{1} \int_{0}^{1} k(t, s) \gamma\left(s, \varphi_{n-1}(s)\right) d s & \\
& +\lambda_{2} \int_{0}^{t} F(t, s) g\left(s, \varphi_{n-1}(s)\right) d s \quad, \quad \phi_{0}(t)=f(t) .
\end{aligned}
$$

Let $V_{s}(t)$ be acontinuous function such that

Then, we obtain

$$
V_{s}(t)=\varphi_{s}(t)-\varphi_{s-1}(t), V_{0}(t)=f(t), \quad \forall s=1,2, \ldots, n .
$$

In view of (2.3), we get

$$
\phi_{n}(t)=\sum_{s=0}^{n} V_{s}(t)
$$

$$
\begin{aligned}
\mu V_{n}(t)=f(t, H( & \left.\left(t, V_{n-1}(t)\right)\right)+\lambda_{1} \int_{0}^{1} k(t, s) \gamma\left(s, V_{n-1}(s)\right) d s \\
& +\lambda_{2} \int_{0}^{t} F(t, s) g\left(s, V_{n-1}(s)\right) d s \quad, \quad\left(V_{0}(t)=f(t)\right) .
\end{aligned}
$$

The normality of Eq. (2.4) takes the form

$$
\begin{aligned}
\left\|V_{n}(t)\right\| \leq \frac{1}{|\mu|}\left\{\left\|f\left(t, H\left(t, V_{n-1}(t)\right)\right)\right\|+\left|\lambda_{1}\right|\left\|\int_{0}^{1}|k(t, s)|\left|\gamma\left(s, V_{n-1}(s)\right)\right| d s\right\|\right. \\
\left.+\left|\lambda_{2}\right|\left\|\int_{0}^{t}|F(t, s)|\left|g\left(s, V_{n-1}(s)\right)\right| d s\right\|\right\} .
\end{aligned}
$$

For $n=1$, we have

$$
\begin{aligned}
\left\|V_{1}(t)\right\| \leq \frac{1}{|\mu|}\left\{\left\|f\left(t, H\left(t, V_{0}(t)\right)\right)\right\|+\left|\lambda_{1}\right|\left\|\int_{0}^{1}|k(t, s)|\left|\gamma\left(s, V_{0}(s)\right)\right| d s\right\|\right. \\
\quad+\left|\lambda_{2}\right|\left\|\int_{0}^{t}\left|F(t, s)\left\|g\left(s, V_{0}(s)\right) \mid d s\right\|\right\} .\right.
\end{aligned}
$$

Using the conditions (i) -(iii), we get

$$
\left\|V_{1}(t)\right\| \leq \alpha G ; \alpha=q\left(1+\left|\lambda_{1}\right| M+\left|\lambda_{2}\right| T S\right) /|\mu|, \quad\left(T=\max _{0 \leq t \leq T} ;\left\|V_{0}(t)\right\|=G\right) .
$$

Therefore, by induction we have

$$
\left\|V_{n}(t)\right\| \leq \alpha^{n} G \quad, n=1,2,3, \ldots .
$$


The last inequality shows that the sequence $\left\{V_{s}(t)\right\}_{s=0}^{\infty}$ is uniformlyconvergent under the condition $\alpha<$ $1 ;$ i.e. $q\left(1+\left|\lambda_{1}\right| M+\left|\lambda_{2}\right| T S\right)<|\mu|$, and hence the sequence $\left\{\varphi_{n}(t)\right\}_{n=0}^{\infty}$ in (2.3) converges uniformly, so we can write

$$
\varphi(t)=\sum_{s=0}^{\infty} V_{s}(t) .(2.7)
$$

The series (2.7) is uniformly convergent to a continuous function $\varphi(t)$, since the terms $V_{s}(t)$ are continuous and domained by $\alpha^{s}$.

Lemma 2:The function $\varphi(t)$ defined by (2.7) is the unique solution of Eq. (1.1).

Proof:Firstly, to show that $\varphi(t)$ is a solution ofEq.(1.1), we set $\varphi(t)=\varphi_{n}(t)+\zeta_{n}(t) ; \zeta_{n}(t) \rightarrow \infty$ as $n \rightarrow \infty$.

From Eq. (2.1), we have

$$
\begin{aligned}
\varphi(t)-\zeta_{n}(t)=\frac{1}{\mu}\left\{f\left(t, H\left(t, \varphi(t)-\zeta_{n}(t)\right)\right)+\lambda_{1} \int_{0}^{1} k(t, s) \gamma\left(s, \varphi(s)-\zeta_{n}(s)\right) d s\right. \\
\left.+\lambda_{2} \int_{0}^{t} F(t, s) g\left(s, \varphi(s)-\zeta_{n}(s)\right) d s\right\} .
\end{aligned}
$$

Using the conditions (i) -(iii), we get

$$
\begin{gathered}
\left\|\mu \varphi(t)-f(t, H(t, \varphi(t)))-\lambda_{1} \int_{0}^{1} k(t, s) \gamma(s, \varphi(s)) d s-\lambda_{2} \int_{0}^{t} F(t, s) g(s, \varphi(s)) d s\right\| \\
\leq\left\|\zeta_{n}(t)\right\|+\alpha\left\|\zeta_{n-1}(t)\right\| .
\end{gathered}
$$

When $n \rightarrow \infty$, we obtain

$\mu \varphi(t)-f(t, H(t, \varphi(t)))-\lambda_{1} \int_{0}^{1} k(t, s) \gamma(s, \varphi(s)) d s-\lambda_{2} \int_{0}^{t} F(t, s) g(s, \varphi(s)) d s=0$.

Hence, $\varphi(t)$ is a solution ofEq.(1.1).

Secondly, to prove that $\varphi(t)$ is a unique solution, we assume that $\tilde{\varphi}(t)$ is another solution of Eq.(1.1), then

$$
\begin{aligned}
\|\varphi(t)-\tilde{\varphi}(t)\| \leq & \frac{1}{|\mu|}\{\|f(t, H(t, \varphi(t)))-f(t, H(t, \tilde{\varphi}(t)))\| \\
& +\left|\lambda_{1}\right|\left\|\int_{0}^{1}|k(t, s)||\gamma(s, \varphi(s))-\gamma(s, \tilde{\varphi}(s))| d s\right\| \\
& \left.+\left|\lambda_{2}\right|\left\|\int_{0}^{t}|F(t, s)||g(s, \varphi(s))-g(s, \tilde{\varphi}(s))| d s\right\|\right\} .
\end{aligned}
$$

In view of the conditions (i) and (ii), we obtain

$\|\varphi(t)-\tilde{\varphi}(t)\| \leq \alpha\|\varphi(t)-\tilde{\varphi}(t)\| ; \alpha=q\left(1+\left|\lambda_{1}\right| M+\left|\lambda_{2}\right| T S\right)<|\mu|$.

Since $\alpha<1$, this can be true if and only if $\varphi(t)=\tilde{\varphi}(t)$; that is, the solution of Eq.(1.1) is unique.

Proof of Theorem1:From lemmas 1and 2 we deduce that thenonlocal H-VIE (1.1)has a unique solution $\varphi(t)$ in the Banach space $C[0, T]$ under the condition $\alpha<1$.

\section{Numerical Methods}

In this section, we discuss the solution of the nonlocal H-VIE (1.1) numerically using two different methodsTrapezoidal rule and Simpson'srule, and determine the error in each method.

\subsection{TheTrapezoidal Rule:}

For using trapezoidal rule, we divide the interval [0,1]into $N$ subintervals with length $h=1 / N, N$ can be even or odd, wheret $=t_{i}, s=s_{j}, 0 \leq i, j \leq N$.Then, Eq.(1.1) reduces to the following nonlocal NAS :

$$
\mu \varphi_{i}=f_{i}\left(H_{i}\left(\varphi_{i}\right)\right)+\lambda_{1} \sum_{j=0}^{N} u_{j} k_{i, j} \gamma_{j}\left(\varphi_{i}\right)+\lambda_{2} \sum_{j=0}^{i} w_{j} F_{i, j} g_{j}\left(\varphi_{i}\right)+R_{N} ; 0 \leq i \leq N .
$$

Where $R_{N}$ is the error of the numerical method and $u_{j}$ and $w_{j}$ are the weights defined by:

$$
u_{j}=\left\{\begin{array}{l}
h / 2 ; j=0, N \\
h \quad ; 0<j<N
\end{array} \quad, \quad w_{j}=\left\{\begin{array}{r}
h \quad ; 0<j<i . j \quad j=0, i \\
0 ; j>i
\end{array}\right.\right.
$$

Also the following notations are used:

$$
\begin{gathered}
\varphi_{i}=\varphi\left(t_{i}\right), f_{i}\left(H_{i}\left(\varphi_{i}\right)\right)=f_{i}\left(t_{i},\left(H_{i}\left(t_{i}, \varphi\left(t_{i}\right)\right)\right)\right), k\left(t_{i}, t_{j}\right)=k_{i, j}, \gamma_{i}\left(\varphi_{i}\right)=\gamma\left(t_{i}, \varphi\left(t_{i}\right)\right), \\
g_{i}\left(\varphi_{i}\right)=g\left(t_{i}, \varphi\left(t_{i}\right)\right) .
\end{gathered}
$$

The formula (3.1) represents $(N+1)$ nonlocal NAS of equations and its solution is the approximation solution ofEq. (1.1).

Definition 1: The estimatetotalerror $R_{N}$ of theTrapezoidal rule is determined by : 


$$
\begin{aligned}
R_{N}=\mid \lambda_{1} \int_{0}^{1} k(t, s) & \gamma(s, \varphi(s)) d s+\lambda_{2} \int_{0}^{t} F(t, s) g(s, \varphi(s)) d s-\lambda_{1} \sum_{j=0}^{N} u_{j} k_{i, j} \gamma_{j}\left(\varphi_{i}\right)-\lambda_{2} \sum_{j=0}^{i} w_{j} F_{i, j} g_{j}\left(\varphi_{i}\right) \mid \\
i & =0,1,2, \ldots, N \\
& =-\frac{1}{12} h^{2} \frac{d^{2}}{d \xi^{2}}\left|\lambda_{1} k\left(t_{N}, \xi\right) \gamma(\xi, \varphi(\xi))+\lambda_{2} F\left(t_{N}, \xi\right) g(\xi, \varphi(\xi))\right|, \xi \in(0,1)
\end{aligned}
$$

\section{-The existence and uniqueness solution of the nonlocal NAS:}

The existence of a unique solution of thenonlocal NAS (3.1), in the space $\ell^{\infty}$, will be proved using Picard's method, under the conditions:

(1)The given sequences $\left\{f_{i}\left(H_{i}\left(\varphi_{i}\right)\right)\right\},\left\{\gamma_{i}\left(\varphi_{i}\right)\right\},\left\{g_{i}\left(\varphi_{i}\right)\right\}$ for all $i$, satisfy fortheconstant $p^{\prime}>\max \left\{p_{m}^{\prime}\right\}$, $m=1,2,3$, the following conditions:

$$
(a)\left|f_{i}\left(H_{i}\left(\varphi_{i}\right)\right)\right| \leq p_{1}^{\prime}\left|\varphi_{i}\right|, \quad(b)\left|\gamma_{i}\left(\varphi_{i}\right)\right| \leq p_{2}^{\prime}\left|\varphi_{i}\right|, \quad(c)\left|g_{i}\left(\varphi_{i}\right)\right| \leq p_{3}^{\prime}\left|\varphi_{i}\right| .
$$

Moreover, for each function $L^{\prime}\left(t_{i}\right)>\max \left\{L_{m}\left(t_{i}\right)\right\} ; m=1,2,3$, and for the constants $\mathcal{L}^{\prime}>\sup _{i}\left|L^{\prime}\left(t_{i}\right)\right|$ and $q^{\prime} \geq \max \left\{p^{\prime}, \mathcal{L}^{\prime}\right\}$, we find

$$
(d)\left|f_{i}\left(H_{i}\left(\varphi_{i}\right)\right)-f_{i}\left(H_{i}\left(\psi_{i}\right)\right)\right| \leq L_{1}\left(t_{i}\right)\left|\varphi_{i}-\psi_{i}\right| . \quad(e)\left|\gamma_{i}\left(\varphi_{i}-\psi_{i}\right)\right| \leq L_{2}\left(t_{i}\right)\left|\varphi_{i}-\psi_{i}\right| .
$$

$(f)\left|g_{i}\left(\varphi_{i}\right)-g_{i}\left(\psi_{i}\right)\right| \leq L_{3}\left(t_{i}\right)\left|\varphi_{i}-\psi_{i}\right|$.

(2) $\sup _{j} \sum_{j=0}^{N}\left|u_{j} k_{i, j}\right| \leq M^{\prime}, \sup _{j} \sum_{j=0}^{N}\left|w_{j} F_{i, j}\right| \leq S^{\prime} \quad,\left(M^{\prime}, S^{\prime}\right.$ are constants $)$.

(3) $\|f\|_{\ell^{\infty}}=\sup _{i}\left|f_{i}\right|=G^{\prime},\left(G^{\prime}\right.$ is a constant $)$.

Theorem 2(without proof): The nonlocal NAS (3.1) has a unique solution in Banach space $\ell^{\infty}$ under the condition: $q^{\prime}\left(1+\left|\lambda_{1}\right| M^{\prime}+\left|\lambda_{2}\right| S^{\prime}\right)<|\mu|$.

In addition, if $N \rightarrow \infty$ then

$$
\left\{\lambda_{1} \sum_{j=0}^{N} u_{j} k_{i, j} \gamma_{j}\left(\varphi_{j}\right)+\lambda_{2} \sum_{j=0}^{i} w_{j} F_{i, j} g_{j}\left(\varphi_{j}\right)\right\} \rightarrow\left\{\lambda_{1} \int_{0}^{1} k(t, s) \gamma(s, \varphi(s)) d s+\lambda_{2} \int_{0}^{t} F(t, s) g(s, \varphi(s)) d s\right\} .
$$

Thus, the solution of thenonlocal NAS (3.1) tends to the solution of the nonlocal H-VIE (1.1).

3.2. Simpson's rule: For using Simpson's rule, we divide the interval $[0,1]$ into $N$ subintervals with length $h=$ $1 / N, N$ is even, where $t=t_{i}, s=t_{j}, 0 \leq i, j \leq N$.Then, the nonlocal NH-VIE (1.1), after approximate the integral terms, reduces to the following nonlocal NAS:

$$
\mu \varphi_{i}=f_{i}\left(H_{i}\left(\varphi_{i}\right)\right)+\lambda_{1} \sum_{j=0}^{N} \rho_{j} k_{i, j} \gamma_{j}\left(\varphi_{j}\right)+\lambda_{2} \sum_{j=0}^{i} \vartheta_{j} F_{i, j} g_{j}\left(\varphi_{j}\right)+\tilde{R}_{N} ; 0 \leq i \leq N .
$$

Where $\tilde{R}_{N}$ is the error of themethod and $\rho_{j}$ and $\vartheta_{j}$ are the weightsgiven by:

$4 h 3,0<j<N$, joddand $\rho j=2 h 3,0<j<N$, jeven.

a) $\left(\rho_{j}=h / 3, j=0, N\right),\left(\rho_{j}=\right.$

b) $\vartheta_{j}$ take the two forms depending on the value of $i$ (odd or even).

1. If $i$ is odd we must use Trapezoidalrule, then $\vartheta_{j}=\widetilde{\omega}_{j} ;\left(\widetilde{\omega}_{j}=h / 2, j=0, i\right),\left(\widetilde{\omega}_{j}=h, 0<j<\right.$ $i$ and $\omega j=0, j>i$.

2. If $i$ is even we must use Simpson's rule, then $\vartheta_{j}=\omega_{j} ;\left(\omega_{j}=h / 3, j=0, i\right),\left(\omega_{j}=4 h / 3 ; 0<j<\right.$ $i$; $j$ odd, $\omega j=2 h 3 ; 0<j<i ; j$ even and $\omega j=0, j>i$.

After neglecting the error in Eq. (3.5)and using the same notations of (3.3), we obtain the following $(N+1)$ nonlocal NAS:

$$
\mu \varphi_{i}=f_{i}\left(H_{i}\left(\varphi_{i}\right)\right)+\lambda_{1} \sum_{j=0}^{N} \rho_{j} k_{i, j} \gamma_{j}\left(\varphi_{i}\right)+\lambda_{2} \sum_{j=0}^{i} \vartheta_{j} F_{i, j} g_{j}\left(\varphi_{i}\right), 0 \leq i \leq N .
$$

Definition 2: The estimate totalerror $\tilde{R}_{N}$ oftheSimpson's rule is determined by :

$$
\begin{gathered}
\tilde{R}_{N}=\left|\lambda_{1} \int_{0}^{1} k(t, s) \gamma(s, \varphi(s)) d s+\lambda_{2} \int_{0}^{t} F(t, s) g(s, \varphi(s)) d s-\lambda_{1} \sum_{j=0}^{N} \rho_{j} k_{i, j} \gamma_{j}\left(\varphi_{j}\right)-\lambda_{2} \sum_{j=0}^{N} \vartheta_{j} F_{i, j} g_{j}\left(\varphi_{j}\right)\right| \\
\quad=-\frac{1}{180} h^{4} \frac{d^{4}}{d \xi^{4}}\left|\lambda_{1} k\left(t_{N}, \xi\right) \gamma(\xi, \varphi(\xi))+\lambda_{2} F\left(t_{N}, \xi\right) g(\xi, \varphi(\xi))\right|, \xi \in(0,1)
\end{gathered}
$$

-The existence and uniqueness solution of the nonlocal NAS: 
In order to guarantee the existence of a unique solution of the nonlocal NAS (3.6) in the space $\ell^{\infty}$, we consider the conditions (1) and (3), and the following condition :

$\left(2^{\prime}\right) \sup _{j} \sum_{j=0}^{N}\left|\rho_{j} k_{i, j}\right| \leq M^{*}, \sup _{j} \sum_{j=0}^{N}\left|\vartheta_{j} v_{i, j}\right| \leq S^{*}\left(M^{*}, S^{*}\right.$ are constants $)$.

Theorem 3(without proof): ThenonlocalNAS (3.6) has a unique solution in the space $\ell^{\infty}$ under the condition: $q^{\prime}\left(1+\left|\lambda_{1}\right| M^{*}+\left|\lambda_{2}\right| S^{*}\right)<|\mu|$.

And if $N \rightarrow \infty$, then

$$
\left\{\lambda_{1} \sum_{j=0}^{N} \rho_{j} k_{i, j} \gamma_{j}\left(\varphi_{j}\right)+\lambda_{2} \sum_{j=0}^{i} \vartheta_{j} F_{i, j} g_{j}\left(\varphi_{j}\right)\right\} \rightarrow\left\{\lambda_{1} \int_{0}^{1} k(t, s) \gamma(s, \varphi(s)) d s+\lambda_{2} \int_{0}^{t} F(t, s) g(s, \varphi(s)) d s\right\} .
$$

Thus, the solution of thenonlocal NAS (3.6) tends to the solution of thenonlocal NH-VIE (1.1).

Theorem 4: If the sequence of continuous functions $\left\{f_{N}(t, H(t, \varphi(t)))\right\}$ converges uniformly to the function $f(t, H(t, \varphi(t)))$ in the space $C[0, T]$, then under the conditions (i) and (ii) of theorem (1), the sequence of approximate solutions $\left\{\varphi_{N}(t)\right\}$ converges uniformly to the exact solution of Eq. (1.1) in the space $C[0, T]$.

Proof:The formula (1.1) with its approximate solution gives

$$
\begin{aligned}
\left|\varphi(t)-\varphi_{N}(t)\right| \leq & \frac{1}{|\mu|}\left\{\left|f(t, H(t, \varphi(t)))-f_{N}(t, H(t, \varphi(t)))\right|\right. \\
& +\left|\lambda_{1}\right| \int_{0}^{1}|k(t, s)|\left|\gamma(s, \varphi(s))-\gamma\left(s, \varphi_{N}(s)\right)\right| d s \\
& \left.+\left|\lambda_{2}\right| \int_{0}^{t}|F(t, s)|\left|g(s, \varphi(s))-g\left(s, \varphi_{N}(s)\right)\right| d s\right\} .
\end{aligned}
$$

Using the conditions (i) and (ii) of theorem (1), we get

$$
\left\|\varphi(t)-\varphi_{N}(t)\right\|_{C[0, T]} \leq \frac{1}{\left(|\mu|-q\left(\left|\lambda_{1}\right| M+\left|\lambda_{2}\right| S T\right)\right)}\left\|f(t, H(t, \varphi(t)))-f_{N}(t, H(t, \varphi(t)))\right\|_{C[0, T]}
$$

Hence, $\left\|\varphi(t)-\varphi_{N}(t)\right\|_{C[0, T]} \rightarrow 0$ since $\left\|f(t, H(t, \varphi(t)))-f_{N}(t, H(t, \varphi(t)))\right\|_{C[0, T]} \rightarrow 0$

as $N \rightarrow \infty$.

Corollary 1: The total error $R_{N}$ of the Trapezoidal rule satisfieslim $\lim _{N \rightarrow \infty} R_{N}=0$.

Proof: From the definition of the $R_{N}$, we have

$$
R_{N}=\left[\varphi_{i}-\left(\varphi_{i}\right)_{N}\right]-\left[\lambda_{1} \sum_{j=0}^{N} u_{j} k_{i, j}\left(\gamma_{j}\left(\varphi_{j}\right)-\gamma_{j}\left(\varphi_{j}\right)_{N}\right)+\lambda_{2} \sum_{j=0}^{N} w_{j} F_{i, j}\left(g_{j}\left(\varphi_{j}\right)-g_{j}\left(\varphi_{j}\right)_{N}\right)\right] \text {. }
$$

The above formula can be adapted in the form

$$
\begin{aligned}
\left|R_{N}\right| \leq \sup _{i} \mid \varphi_{i}- & \left(\varphi_{i}\right)_{N}|+| \lambda_{1}\left|\sup _{j} \sum_{j=0}^{N}\right| u_{j} k_{i, j}\left|\sup _{j}\right|\left(\gamma_{j}\left(\varphi_{j}\right)-\gamma_{j}\left(\varphi_{j}\right)_{N}\right) \mid \\
& \left.+\left|\lambda_{2}\right| \sup _{j} \sum_{j=0}^{N}\left|w_{j} F_{i, j}\right| \sup _{j} \mid g_{j}\left(\varphi_{j}\right)-g_{j}\left(\varphi_{j}\right)\right)_{N} \mid .
\end{aligned}
$$

In view of conditions (1) and (2), we get

$$
\left|R_{N}\right| \leq\left(1+\left|\lambda_{1}\right| M^{\prime} q^{\prime}+\left|\lambda_{2}\right| S^{\prime} q^{\prime}\right)\left\|\varphi\left(t_{i}\right)-\varphi_{N}\left(t_{i}\right)\right\|_{\ell^{\infty}}, \quad \forall N
$$

Since each term $R_{N}$ is bounded above, hence for $t=t_{i}$, we deduce

$$
\left\|R_{N}\right\|_{\ell^{\infty}} \leq\left(1+\left|\lambda_{1}\right| M^{\prime} q^{\prime}+\left|\lambda_{2}\right| S^{\prime} q^{\prime}\right)\left\|\varphi(t)-\varphi_{N}(t)\right\|_{C[0, T]} .
$$

Since $\left\|\varphi(t)-\varphi_{N}(t)\right\|_{C[0, T]} \rightarrow 0$ as $N \rightarrow \infty$, thenlim $\lim _{N \rightarrow \infty}\left\|R_{N}\right\|_{\ell^{\infty}}=0$.

Corollary 2(without proof): The total error $\widetilde{R}_{N}$ of theSimpson's rule satisfies $\lim _{N \rightarrow \infty} \tilde{R}_{N}=0$.

For thenonlocal H-VIE:

\section{Numerical Examples}

$$
\begin{aligned}
\mu \varphi(t)=f(t, H(t, \varphi(t)))+0.01 \int_{0}^{1} t s^{2} \gamma(s, \varphi(s)) d s & \\
& +0.01 \int_{0}^{t} t s g(s, \varphi(s)) d s,\left(\varphi(t)=t^{2}\right)\left(\lambda_{1}=\lambda_{2}=0.01,0 \leq t \leq T<1\right),
\end{aligned}
$$


Trapezoidal method and Simpson's method will be usedto obtain the numerical solution for the nonlocal H-VI (4.1) for different values of $h$ and $\mu$ for several forms of $H(t, \varphi(t)), \gamma(s, \varphi(s)), g(s, \varphi(s))$, as shown in the following:

Case (I) when there is no memory term $(H(t, \varphi(t))=0)$.

Here we solve, numerically (4.1) for different value of $(\mu=0.1,0.5,1)$, and $h=0.625$.

(Case I.1) When there is no memory term $(H(t, \varphi(t))=0)$ and for the nonlinear functions $\gamma(s, \varphi(s))=$ $s \varphi^{2}(s), g(s, \varphi(s))=\varphi^{2}(s)$.

Table(1)

\begin{tabular}{|c|c|c|c|c|c|c|c|}
\hline \multicolumn{8}{|c|}{ Case I.1: Trapezoidal method $H(t, \varphi(t))=0, \gamma(s, \varphi(s))=s \varphi^{2}(s), g(s, \varphi(s))=\varphi^{2}(s)$} \\
\hline \multirow[t]{2}{*}{$t$} & \multirow[t]{2}{*}{$\varphi$} & \multicolumn{2}{|c|}{$\mu=0.1, h=0.625, N=16$} & \multicolumn{2}{|c|}{$\mu=0.5, h=0.625, N=16$} & \multicolumn{2}{|c|}{$\mu=1, h=0.625, N=16$} \\
\hline & & $\varphi^{T r}$ & $E^{T r}$ & $\varphi^{T r}$ & $E^{T r}$ & $\varphi^{T r}$ & $E^{T r}$ \\
\hline 0 & $0.00000 \mathrm{E}+00$ & $0.00000 \mathrm{E}+00$ & $0.00000 \mathrm{E}+00$ & $0.00000 \mathrm{E}+00$ & $0.00000 \mathrm{E}+00$ & $0.00000 \mathrm{E}+00$ & $0.00000 \mathrm{E}+00$ \\
\hline 0.25 & $6.25000 \mathrm{E}-02$ & $6.25596 \mathrm{E}-02$ & $5.96000 \mathrm{E}-05$ & $6.25115 \mathrm{E}-02$ & $1.15000 \mathrm{E}-05$ & $6.25057 \mathrm{E}-02$ & 5.70000E-06 \\
\hline 0.5 & $2.50000 \mathrm{E}-01$ & $2.50124 \mathrm{E}-01$ & $1.24000 \mathrm{E}-04$ & $2.50024 \mathrm{E}-01$ & $2.40000 \mathrm{E}-05$ & $2.50012 \mathrm{E}-01$ & $1.20000 \mathrm{E}-05$ \\
\hline 0.75 & $5.62500 \mathrm{E}-01$ & $5.62719 \mathrm{E}-01$ & $2.19000 \mathrm{E}-04$ & $5.62542 \mathrm{E}-01$ & 4.20000E-05 & $5.62521 \mathrm{E}-01$ & $2.10000 \mathrm{E}-05$ \\
\hline 1 & $1.00000 \mathrm{E}+00$ & $1.00041 \mathrm{E}+00$ & $4.10000 \mathrm{E}-04$ & $1.00008 \mathrm{E}+00$ & $8.00000 \mathrm{E}-05$ & $1.00004 \mathrm{E}+00$ & $4.00000 \mathrm{E}-05$ \\
\hline
\end{tabular}

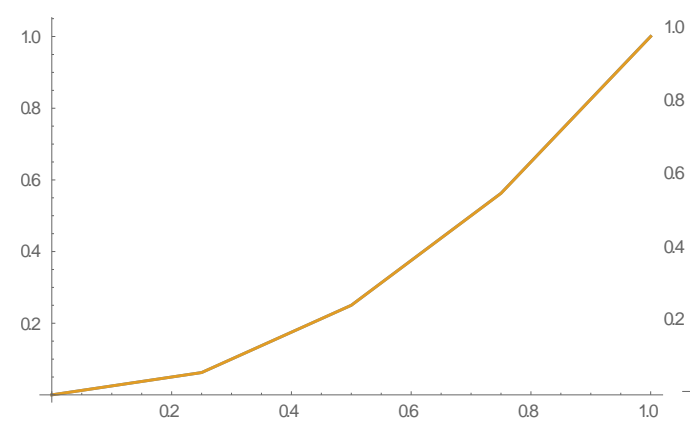

Fig.(1-i) $\mu=0.1, h=0.625$

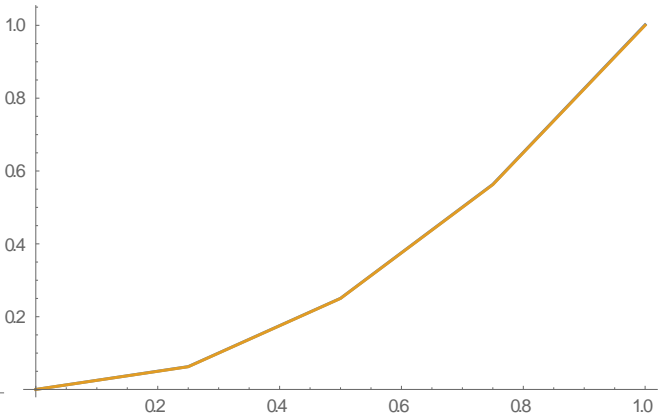

Fig.(1-ii) $\mu=0.5, h=0.625$

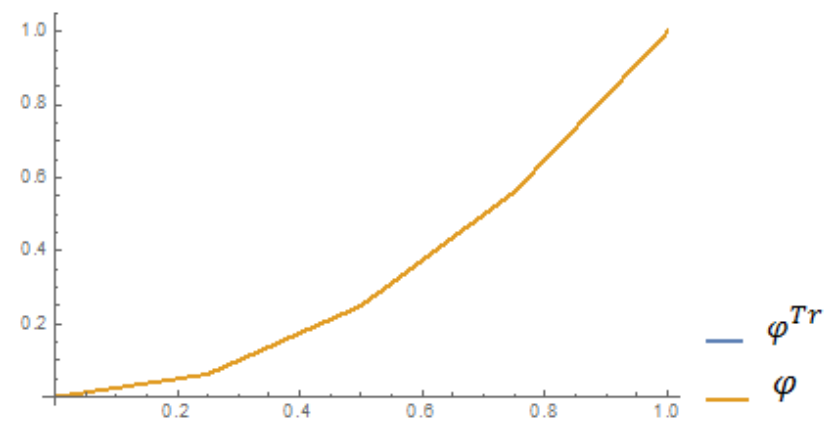

Fig.(1-iii) $\mu=1, h=0.625$

-Figs. (1), describethe relation between the exact solution and numerical solution, when $H(t, \varphi(t))=$ $0, \gamma(s, \varphi(s))=s \varphi^{2}(s), g(s, \varphi(s))=\varphi^{2}(s)$, using Trapezoidal method, with $(\lambda=0.1, h=0.625$ and $N=$ 16at $\mu=0.1$ in Fig. (1.i), $\mu=0.51 \mathrm{n}$ Fig (1.ii) and $\mu=1$ in Fig. (1.iii)

Table(2)

\begin{tabular}{|l|c|c|c|c|c|c|c|}
\hline \multicolumn{2}{|c|}{ Case I.1: Simpson's method $H(t, \varphi(t))=0, \gamma(s, \varphi(s))=s \varphi^{2}(s), g(s, \varphi(s))=\varphi^{2}(s)$} \\
\hline \multirow{2}{*}{$t$} & $\varphi$ & $\mu=0.1, h=0.625, N=16$ & $\mu=0.5, h=0.625, N=16$ & $\mu=1, h=0.625, N=16$ \\
\cline { 3 - 8 } & & $\varphi^{S}$ & $E^{S}$ & $\varphi^{S}$ & $E^{S}$ & $\varphi^{S}$ & $E^{S}$ \\
\hline 0 & $0.00000 \mathrm{E}+00$ & $0.00000 \mathrm{E}+00$ & $0.00000 \mathrm{E}+00$ & $0.00000 \mathrm{E}+00$ & $0.00000 \mathrm{E}+00$ & $0.00000 \mathrm{E}+00$ & $0.00000 \mathrm{E}+00$ \\
\hline 0.25 & $6.25000 \mathrm{E}-02$ & $6.25009 \mathrm{E}-02$ & $9.44354 \mathrm{E}-07$ & $6.25001 \mathrm{E}-02$ & $1.09159 \mathrm{E}-07$ & $6.25000 \mathrm{E}-02$ & $4.97946 \mathrm{E}-08$ \\
\hline 0.5 & $2.50000 \mathrm{E}-01$ & $2.50002 \mathrm{E}-01$ & $1.94062 \mathrm{E}-06$ & $2.50000 \mathrm{E}-01$ & $2.27986 \mathrm{E}-07$ & $2.50000 \mathrm{E}-01$ & $1.04389 \mathrm{E}-07$ \\
\hline 0.75 & $5.62500 \mathrm{E}-01$ & $5.62503 \mathrm{E}-01$ & $3.17605 \mathrm{E}-06$ & $5.62500 \mathrm{E}-01$ & $3.71245 \mathrm{E}-07$ & $5.62500 \mathrm{E}-01$ & $1.69848 \mathrm{E}-07$ \\
\hline 1 & $1.00000 \mathrm{E}+00$ & $1.00001 \mathrm{E}+00$ & $6.74107 \mathrm{E}-06$ & $1.00000 \mathrm{E}+00$ & $6.27828 \mathrm{E}-07$ & $1.00000 \mathrm{E}+00$ & $2.70709 \mathrm{E}-07$ \\
\hline
\end{tabular}




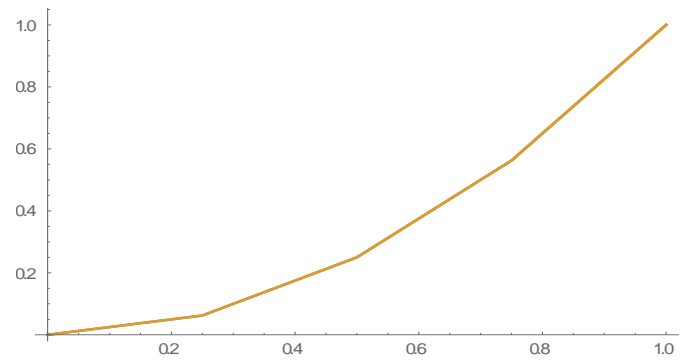

Fig.(2-i) $\mu=0.1, h=0.625$

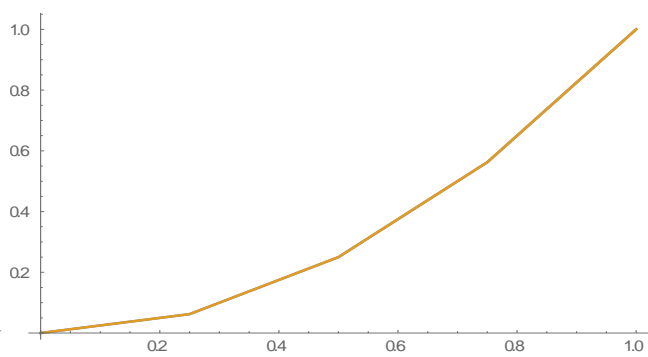

Fig.(2-ii) $\mu=0.5, h=0.625$

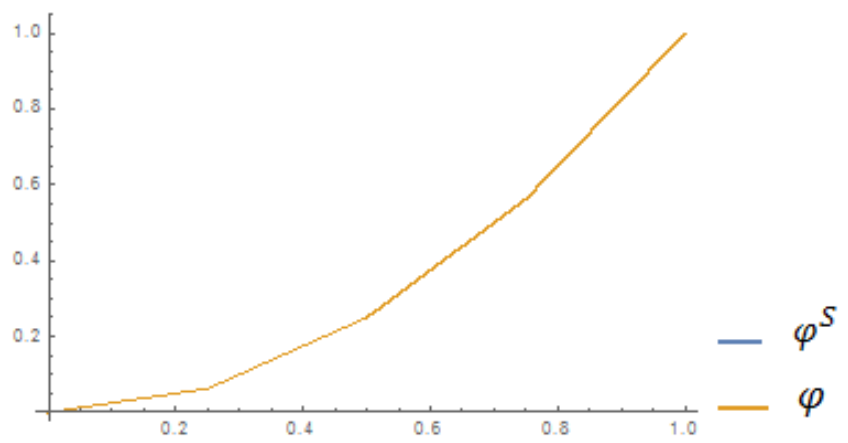

Fig.(2-iii) $\mu=1, h=0.625$

- Figs. (2), Describe the relation between the exact solution and numerical solution, when $H(t, \varphi(t))=$ $0, \gamma(s, \varphi(s))=s \varphi^{2}(s), g(s, \varphi(s))=\varphi^{2}(s)$, using Simpson'smethod, and $h=0.625, \lambda_{1,2}=0.01, N=$ 16 at $\mu=0.1$ in Fig. (2.i), $\mu=0.5$ in Fig (2.ii) and $\mu=1$ in Fig. (2.iii)

(Case I.2) Whenthere is no memory term;i.e. $H(t, \varphi(t))=0, \gamma(s, \varphi(s))=s \varphi^{2}(s)$, while $g(s, \varphi(s))=$ $\varphi(s)$.

Table(3)

\begin{tabular}{|c|c|c|c|c|c|c|c|}
\hline \multicolumn{2}{|c|}{ Case I.2 : Trapezoidal method $H(t, \varphi(t))=0, \gamma(s, \varphi(s))=s \varphi^{2}(s), g(s, \varphi(s))=\varphi(s)$} \\
\hline \multirow{2}{*}{$t$} & $\varphi$ & $\mu=0.1, h=0.625, N=16$ & $\mu=0.5, h=0.625, N=16$ & \multicolumn{2}{c|}{$\mu=1, h=0.625, N=16$} \\
\cline { 3 - 8 } & & $\varphi^{T r}$ & $E^{T r}$ & $\varphi^{T r}$ & $E^{T r}$ & $\varphi^{T r}$ & $E^{T r}$ \\
\hline 0 & $0.00000 \mathrm{E}+00$ & $0.00000 \mathrm{E}+00$ & $0.00000 \mathrm{E}+00$ & $0.00000 \mathrm{E}+00$ & $0.00000 \mathrm{E}+00$ & $0.00000 \mathrm{E}+00$ & $0.00000 \mathrm{E}+00$ \\
\hline 0.25 & $6.25000 \mathrm{E}-02$ & $6.25607 \mathrm{E}-02$ & $6.07000 \mathrm{E}-05$ & $6.25118 \mathrm{E}-02$ & $1.18000 \mathrm{E}-05$ & $6.25059 \mathrm{E}-02$ & $5.90000 \mathrm{E}-06$ \\
\hline 0.5 & $2.50000 \mathrm{E}-01$ & $2.50131 \mathrm{E}-01$ & $1.31000 \mathrm{E}-04$ & $2.50025 \mathrm{E}-01$ & $2.50000 \mathrm{E}-05$ & $2.50013 \mathrm{E}-01$ & $1.30000 \mathrm{E}-05$ \\
\hline 0.75 & $5.62500 \mathrm{E}-01$ & $5.62722 \mathrm{E}-01$ & $2.22000 \mathrm{E}-04$ & $5.62543 \mathrm{E}-01$ & $4.30000 \mathrm{E}-05$ & $5.62521 \mathrm{E}-01$ & $2.10000 \mathrm{E}-05$ \\
\hline 1 & $1.00000 \mathrm{E}+00$ & $1.00034 \mathrm{E}+00$ & $3.40000 \mathrm{E}-04$ & $1.00007 \mathrm{E}+00$ & $7.00000 \mathrm{E}-05$ & $1.00003 \mathrm{E}+00$ & $3.00000 \mathrm{E}-05$ \\
\hline
\end{tabular}

-Table.(3), describe the relation between the exact solution and numerical solution, when $H(t, \varphi(t))=$ $0, \gamma(s, \varphi(s))=s \varphi^{2}(s), g(s, \varphi(s))=\varphi(s)$, using Trapezoidal method.

Table(4)

\begin{tabular}{|c|c|c|c|c|c|c|c|}
\hline \multicolumn{2}{|c|}{ Case I.2 Simpson's method $H(t, \varphi(t))=0, \gamma(s, \varphi(s))=s \phi^{2}(s), g(s, \varphi(s))=\varphi(s)$} \\
\hline \multirow{2}{*}{$t$} & $\varphi$ & $\mu=0.1, h=0.625, N=16$ & $\mu=0.5, h=0.625, N=16$ & \multicolumn{2}{c|}{$\mu=1, h=0.625, N=16$} \\
\cline { 3 - 8 } & & $\varphi^{S}$ & $E^{S}$ & $\varphi^{S}$ & $E^{S}$ & $\varphi^{S}$ & $E^{S}$ \\
\hline 0 & $0.00000 \mathrm{E}+00$ & $0.00000 \mathrm{E}+00$ & $0.00000 \mathrm{E}+00$ & $0.00000 \mathrm{E}+00$ & $0.00000 \mathrm{E}+00$ & $0.00000 \mathrm{E}+00$ & $0.00000 \mathrm{E}+00$ \\
\hline 0.25 & $6.25000 \mathrm{E}-02$ & $6.25001 \mathrm{E}-02$ & $1.36974 \mathrm{E}-07$ & $6.25001 \mathrm{E}-02$ & $6.24776 \mathrm{E}-08$ & $6.25000 \mathrm{E}-02$ & $3.69302 \mathrm{E}-08$ \\
\hline 0.5 & $2.50000 \mathrm{E}-01$ & $2.50000 \mathrm{E}-01$ & $1.60540 \mathrm{E}-07$ & $2.50000 \mathrm{E}-01$ & $9.39613 \mathrm{E}-08$ & $2.50000 \mathrm{E}-01$ & $6.16553 \mathrm{E}-08$ \\
\hline 0.75 & $5.62500 \mathrm{E}-01$ & $5.62500 \mathrm{E}-01$ & $1.73337 \mathrm{E}-07$ & $5.62500 \mathrm{E}-01$ & $1.14231 \mathrm{E}-07$ & $5.62500 \mathrm{E}-01$ & $8.00044 \mathrm{E}-08$ \\
\hline 1 & $1.00000 \mathrm{E}+00$ & $1.00000 \mathrm{E}+00$ & $1.87988 \mathrm{E}-07$ & $1.00000 \mathrm{E}+00$ & $1.31819 \mathrm{E}-07$ & $1.00000 \mathrm{E}+00$ & $9.60355 \mathrm{E}-08$ \\
\hline
\end{tabular}

-Table. (4), describe the relation between the exact solution and numerical solution, when $H(t, \varphi(t))=$ $0, \gamma(s, \varphi(s))=s \phi^{2}(s), g(s, \varphi(s))=\varphi(s)$, using Simpson'smethod.

Case (II) When $H(t, \varphi(t))$ takes a linear form $(H(t, \varphi(t))=t \varphi(t))$.

Here we solve, numerically $(4.1)$ for different value of $(h=0.25,0.125,0.625)$ at $\mu=0.001, \lambda_{1,2}=0.01$. 
Table(5)

\begin{tabular}{|l|c|c|c|c|c|c|c|}
\hline \multicolumn{2}{|c|}{ CaseII.1Trapezoidal method $H(t, \varphi(t))=t \varphi(t), \gamma(s, \varphi(s))=s \phi^{2}(s), g(s, \varphi(s))=\varphi^{2}(s)$} \\
\hline \multirow{2}{*}{$t$} & $\varphi$ & \multicolumn{2}{|c|}{$h=0.25, N=4$} & \multicolumn{2}{c|}{$h=0.125, N=8$} & \multicolumn{2}{c|}{$h=0.625, N=16$} \\
\cline { 3 - 8 } & & $\varphi^{T r}$ & $E^{T r}$ & $\varphi^{T r}$ & $E^{T r}$ & $\varphi^{T r}$ & $E^{T r}$ \\
\hline 0 & $0.00000 \mathrm{E}+00$ & $0.00000 \mathrm{E}+00$ & $0.00000 \mathrm{E}+00$ & $0.00000 \mathrm{E}+00$ & $0.00000 \mathrm{E}+00$ & $0.00000 \mathrm{E}+00$ & $0.00000 \mathrm{E}+00$ \\
\hline 0.25 & $6.25000 \mathrm{E}-02$ & $6.28549 \mathrm{E}-02$ & $3.54916 \mathrm{E}-04$ & $6.25908 \mathrm{E}-02$ & $9.07780 \mathrm{E}-05$ & $6.25228 \mathrm{E}-02$ & $2.28245 \mathrm{E}-05$ \\
\hline 0.5 & $2.50000 \mathrm{E}-01$ & $2.50370 \mathrm{E}-01$ & $3.70374 \mathrm{E}-04$ & $2.50095 \mathrm{E}-01$ & $9.47568 \mathrm{E}-05$ & $2.50024 \mathrm{E}-01$ & $2.38264 \mathrm{E}-05$ \\
\hline 0.75 & $5.62500 \mathrm{E}-01$ & $5.62936 \mathrm{E}-01$ & $4.36213 \mathrm{E}-04$ & $5.62611 \mathrm{E}-01$ & $1.11400 \mathrm{E}-04$ & $5.62528 \mathrm{E}-01$ & $2.79985 \mathrm{E}-05$ \\
\hline 1 & $1.00000 \mathrm{E}+00$ & $1.00061 \mathrm{E}+00$ & $6.14772 \mathrm{E}-04$ & $1.00016 \mathrm{E}+00$ & $1.56278 \mathrm{E}-04$ & $1.00004 \mathrm{E}+00$ & $3.92329 \mathrm{E}-05$ \\
\hline
\end{tabular}

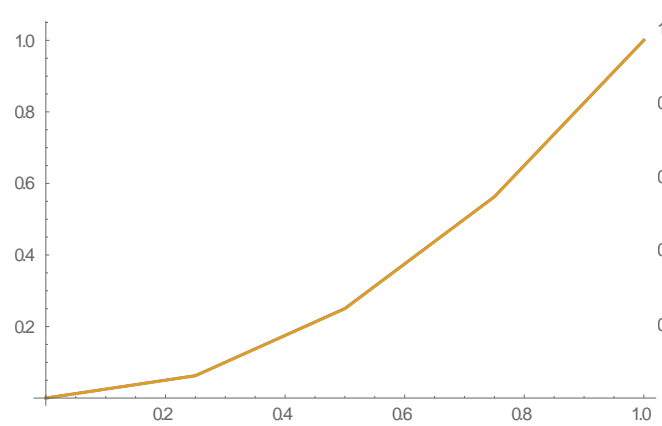

Fig.(5-i) $h=0.25, N=4$

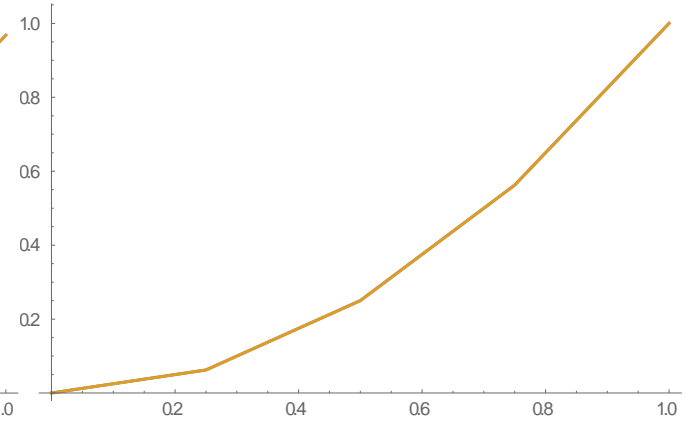

Fig.(5-ii) $h=0.125, N=8$

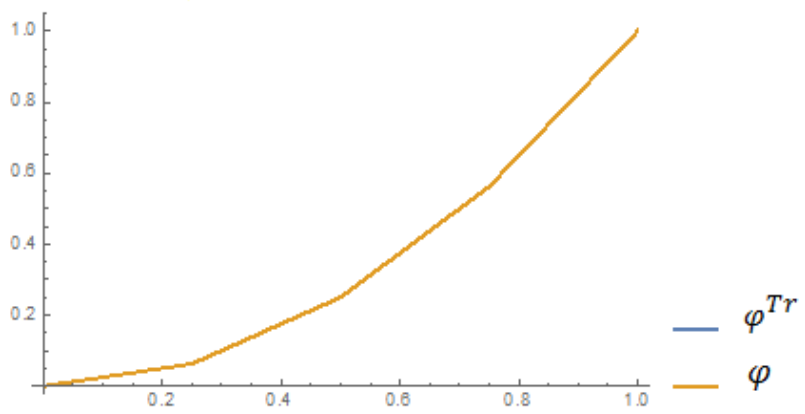

Fig.(5-iii) $h=0.625, N=16$

- Figs. (5) describe the relation between the exact solution and numerical solution, when $H(t, \varphi(t))=$ $t \varphi(t), \gamma(s, \varphi(s))=s \varphi^{2}(s), g(s, \varphi(s))=\phi^{2}(s)$, using Trapezoidal method, with $\lambda=0.01, \mu=0.001$, at $h=0.25,(N=4) ; h=0.125,(N=8) ; h=0.625(N=14)$ in Fig. (5.i), Fig (5.ii) and Fig.(5.iii), respectively.

Table(6)

\begin{tabular}{|c|c|c|c|c|c|c|c|}
\hline \multicolumn{2}{|c|}{ CaseII.1 Simpson's method $H(t, \varphi(t))=t \varphi(t), \gamma(s, \varphi(s))=s \varphi^{2}(s), g(s, \varphi(s))=\varphi^{2}(s)$} \\
\hline \multirow{2}{*}{$t$} & $\varphi$ & \multicolumn{2}{|c|}{$h=0.25, N=4$} & \multicolumn{2}{c|}{$h=0.125, N=8$} & \multicolumn{2}{c|}{$h=0.625, N=16$} \\
\cline { 3 - 8 } & & $\varphi^{S}$ & $E^{S}$ & $\varphi^{S}$ & $E^{S}$ & $\varphi^{S}$ & $E^{S}$ \\
\hline 0 & $0.00000 \mathrm{E}+00$ & $0.00000 \mathrm{E}+00$ & $0.00000 \mathrm{E}+00$ & $0.00000 \mathrm{E}+00$ & $0.00000 \mathrm{E}+00$ & $0.00000 \mathrm{E}+00$ & $0.00000 \mathrm{E}+00$ \\
\hline 0.25 & $6.25000 \mathrm{E}-02$ & $6.25424 \mathrm{E}-02$ & $4.24402 \mathrm{E}-05$ & $6.25029 \mathrm{E}-02$ & $2.90269 \mathrm{E}-06$ & $6.25002 \mathrm{E}-02$ & $2.00566 \mathrm{E}-07$ \\
\hline 0.5 & $2.50000 \mathrm{E}-01$ & $2.50045 \mathrm{E}-01$ & $4.49736 \mathrm{E}-05$ & $2.50003 \mathrm{E}-01$ & $3.06184 \mathrm{E}-06$ & $2.50000 \mathrm{E}-01$ & $2.10634 \mathrm{E}-07$ \\
\hline 0.75 & $5.62500 \mathrm{E}-01$ & $5.62622 \mathrm{E}-01$ & $1.22358 \mathrm{E}-04$ & $5.62503 \mathrm{E}-01$ & $3.32967 \mathrm{E}-06$ & $5.62500 \mathrm{E}-01$ & $2.29351 \mathrm{E}-07$ \\
\hline 1 & $1.00000 \mathrm{E}+00$ & $1.00006 \mathrm{E}+00$ & $5.52195 \mathrm{E}-05$ & $1.00000 \mathrm{E}+00$ & $3.78196 \mathrm{E}-06$ & $1.00000 \mathrm{E}+00$ & $2.75956 \mathrm{E}-07$ \\
\hline
\end{tabular}

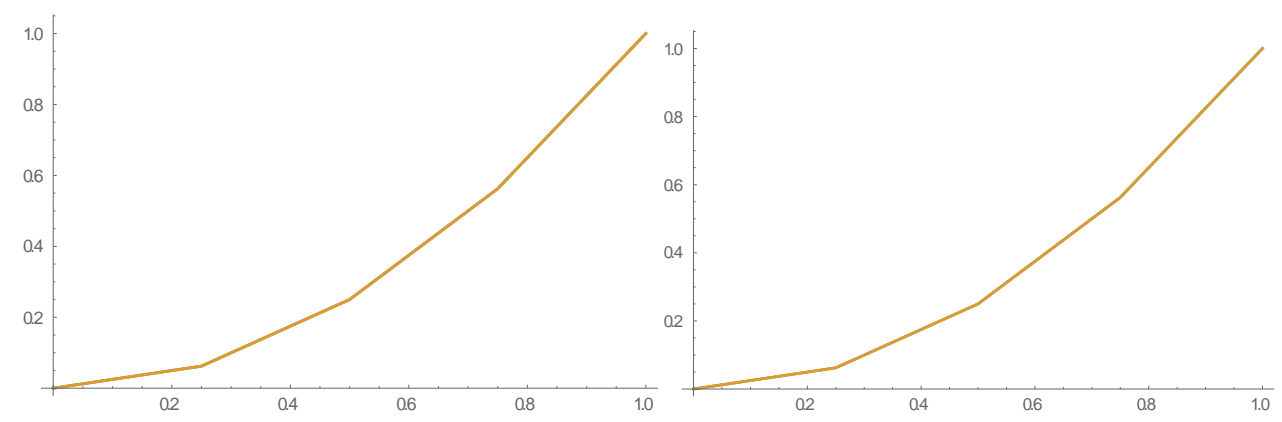

Fig.(6-i) $h=0.25, N=$ 4Fig. (6-ii) $h=0.125, N=8$ 


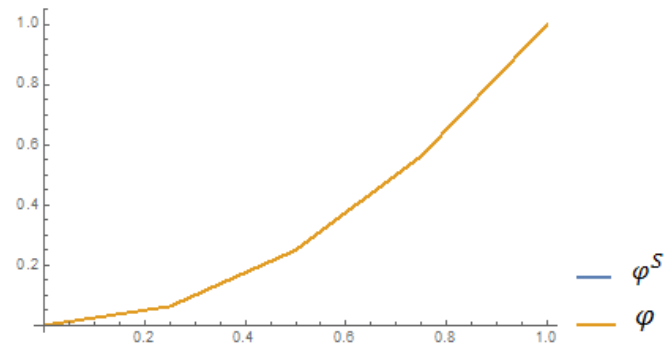

Fig.(6-iii) $h=0.625, N=16$

-Figs. (6) describe the relation between the exact solution and numerical solution, when

$H(t, \varphi(t))=t \varphi(t), \gamma(s, \varphi(s))=s \varphi^{2}(s), g(s, \varphi(s))=\varphi^{2}(s)$, using Simpson's method, with $\lambda=0.01, \mu=$ 0.001 at $h=0.25, N=4 ; h=0.125, N=8 ; h=0.625, N=16$ in Fig. (6.i), Fig (6.ii) and Fig. (6.iii), respectively.

(II.2) When the memory in a linear term $(H(t, \varphi(t))=t \varphi(t))$.

Table(7)

\begin{tabular}{|c|c|c|c|c|c|c|c|}
\hline \multicolumn{2}{|c|}{ CaseII.2 Trapezoidal method $H(t, \varphi(t))=t \varphi(t), \gamma(s, \varphi(s))=s \varphi^{2}(s), g(s, \varphi(s))=\varphi(s)$} \\
\hline \multirow{2}{*}{$t$} & $\varphi$ & \multicolumn{2}{|c|}{$h=0.25, N=4$} & \multicolumn{2}{c|}{$h=0.125, N=8$} & \multicolumn{2}{c|}{$h=0.625, N=16$} \\
\cline { 3 - 8 } & & $\varphi^{T r}$ & $E^{T r}$ & $\varphi^{T r}$ & $E^{T r}$ & $\varphi^{T r}$ & $E^{T r}$ \\
\hline 0 & $0.00000 \mathrm{E}+00$ & $0.00000 \mathrm{E}+00$ & $0.00000 \mathrm{E}+00$ & $0.00000 \mathrm{E}+00$ & $0.00000 \mathrm{E}+00$ & $0.00000 \mathrm{E}+00$ & $0.00000 \mathrm{E}+00$ \\
\hline 0.25 & $6.25000 \mathrm{E}-02$ & $6.28637 \mathrm{E}-02$ & $3.63691 \mathrm{E}-04$ & $6.25930 \mathrm{E}-02$ & $9.29556 \mathrm{E}-05$ & $6.25234 \mathrm{E}-02$ & $2.33680 \mathrm{E}-05$ \\
\hline 0.5 & $2.50000 \mathrm{E}-01$ & $2.50394 \mathrm{E}-01$ & $3.94015 \mathrm{E}-04$ & $2.50101 \mathrm{E}-01$ & $1.00542 \mathrm{E}-04$ & $2.50025 \mathrm{E}-01$ & $2.52649 \mathrm{E}-05$ \\
\hline 0.75 & $5.62500 \mathrm{E}-01$ & $5.62944 \mathrm{E}-01$ & $4.43702 \mathrm{E}-04$ & $5.62613 \mathrm{E}-01$ & $1.12966 \mathrm{E}-04$ & $5.62528 \mathrm{E}-01$ & $2.83713 \mathrm{E}-05$ \\
\hline 1 & $1.00000 \mathrm{E}+00$ & $1.00051 \mathrm{E}+00$ & $5.13197 \mathrm{E}-04$ & $1.00013 \mathrm{E}+00$ & $1.30343 \mathrm{E}-04$ & $1.00003 \mathrm{E}+00$ & $3.27157 \mathrm{E}-05$ \\
\hline
\end{tabular}

Table(8)

\begin{tabular}{|c|c|c|c|c|c|c|c|}
\hline \multicolumn{2}{|c|}{ Case II.2 : Simpson's method $H(t, \varphi(t))=t \varphi(t), \gamma(s, \varphi(s))=s \varphi^{2}(s), g(s, \varphi(s))=\varphi(s)$} \\
\hline \multirow{2}{*}{$t$} & $\varphi$ & \multicolumn{2}{c|}{$h=0.25, N=4$} & \multicolumn{2}{c|}{$h=0.125, N=8$} & \multicolumn{2}{c|}{$h=0.625, N=16$} \\
\cline { 3 - 8 } & & $\varphi^{S}$ & $E^{S}$ & $\varphi^{S}$ & $E^{S}$ & $\varphi^{S}$ & $E^{S}$ \\
\hline 0 & $0.00000 \mathrm{E}+00$ & $0.00000 \mathrm{E}+00$ & $0.00000 \mathrm{E}+00$ & $0.00000 \mathrm{E}+00$ & $0.00000 \mathrm{E}+00$ & $0.00000 \mathrm{E}+00$ & $0.00000 \mathrm{E}+00$ \\
\hline 0.25 & $6.25000 \mathrm{E}-02$ & $6.25514 \mathrm{E}-02$ & $5.13603 \mathrm{E}-05$ & $6.25028 \mathrm{E}-02$ & $2.84008 \mathrm{E}-06$ & $6.25002 \mathrm{E}-02$ & $1.92717 \mathrm{E}-07$ \\
\hline 0.5 & $2.50000 \mathrm{E}-01$ & $2.50042 \mathrm{E}-01$ & $4.17608 \mathrm{E}-05$ & $2.50003 \mathrm{E}-01$ & $2.85183 \mathrm{E}-06$ & $2.50000 \mathrm{E}-01$ & $1.94208 \mathrm{E}-07$ \\
\hline 0.75 & $5.62500 \mathrm{E}-01$ & $5.62630 \mathrm{E}-01$ & $1.29708 \mathrm{E}-04$ & $5.62503 \mathrm{E}-01$ & $2.87404 \mathrm{E}-06$ & $5.62500 \mathrm{E}-01$ & $1.98724 \mathrm{E}-07$ \\
\hline 1 & $1.00000 \mathrm{E}+00$ & $1.00004 \mathrm{E}+00$ & $4.21790 \mathrm{E}-05$ & $1.00000 \mathrm{E}+00$ & $2.92484 \mathrm{E}-06$ & $1.00000 \mathrm{E}+00$ & $2.10280 \mathrm{E}-07$ \\
\hline
\end{tabular}

(II.3) When the memory in a linear term $(H(t, \varphi(t))=t \varphi(t))$ and the function Fredholm is linear $\gamma(s, \varphi(s))=$ $\varphi(s)$ and the function of Volterra is nonlinear $g(s, \varphi(s))=\varphi^{2}(s)$.

Table(9)

\begin{tabular}{|c|c|c|c|c|c|c|c|}
\hline \multicolumn{2}{|c|}{ CaseII.3 Trapezoidal method $H(t, \varphi(t))=t \varphi(t), \gamma(s, \varphi(s))=\varphi(s), g(s, \varphi(s))=\varphi^{2}(s)$} \\
\hline \multirow{2}{*}{$t$} & $\varphi$ & \multicolumn{2}{|c|}{$h=0.25, N=4$} & \multicolumn{2}{c|}{$h=0.125, N=8$} & \multicolumn{2}{c|}{$h=0.625, N=16$} \\
\cline { 3 - 8 } & & $\varphi^{T r}$ & $E^{T r}$ & $\varphi^{T r}$ & $E^{T r}$ & $\varphi^{T r}$ & $E^{T r}$ \\
\hline 0 & $0.00000 \mathrm{E}+00$ & $0.00000 \mathrm{E}+00$ & $0.00000 \mathrm{E}+00$ & $0.00000 \mathrm{E}+00$ & $0.00000 \mathrm{E}+00$ & $0.00000 \mathrm{E}+00$ & $0.00000 \mathrm{E}+00$ \\
\hline 0.25 & $6.25000 \mathrm{E}-02$ & $6.27082 \mathrm{E}-02$ & $2.08182 \mathrm{E}-04$ & $6.25523 \mathrm{E}-02$ & $5.23091 \mathrm{E}-05$ & $6.25131 \mathrm{E}-02$ & $1.30937 \mathrm{E}-05$ \\
\hline 0.5 & $2.50000 \mathrm{E}-01$ & $2.50223 \mathrm{E}-01$ & $2.23295 \mathrm{E}-04$ & $2.50056 \mathrm{E}-01$ & $5.61993 \mathrm{E}-05$ & $2.50014 \mathrm{E}-01$ & $1.40733 \mathrm{E}-05$ \\
\hline 0.75 & $5.62500 \mathrm{E}-01$ & $5.62789 \mathrm{E}-01$ & $2.88835 \mathrm{E}-04$ & $5.62573 \mathrm{E}-01$ & $7.27665 \mathrm{E}-05$ & $5.62518 \mathrm{E}-01$ & $1.82264 \mathrm{E}-05$ \\
\hline 1 & $1.00000 \mathrm{E}+00$ & $1.00047 \mathrm{E}+00$ & $4.66820 \mathrm{E}-04$ & $1.00012 \mathrm{E}+00$ & $1.17498 \mathrm{E}-04$ & $1.00003 \mathrm{E}+00$ & $2.94241 \mathrm{E}-05$ \\
\hline
\end{tabular}

Table(10)

\begin{tabular}{|c|c|c|c|c|c|c|c|}
\hline \multicolumn{2}{|c|}{ CaseII.3Simpson's method $H(t, \varphi(t))=t \varphi(t), \gamma(s, \varphi(s))=s \varphi(s), g(s, \varphi(s))=\varphi^{2}(s)$} \\
\hline \multirow{2}{*}{$t$} & $\varphi$ & \multicolumn{2}{c|}{$h=0.25, N=4$} & \multicolumn{2}{c|}{$h=0.125, N=8$} & \multicolumn{2}{c|}{$h=0.625, N=16$} \\
\cline { 3 - 8 } & & $\varphi^{S}$ & $E^{S}$ & $\varphi^{S}$ & $E^{S}$ & $\varphi^{S}$ & $E^{S}$ \\
\hline 0 & $0.00000 \mathrm{E}+00$ & $0.00000 \mathrm{E}+00$ & $0.00000 \mathrm{E}+00$ & $0.00000 \mathrm{E}+00$ & $0.00000 \mathrm{E}+00$ & $0.00000 \mathrm{E}+00$ & $0.00000 \mathrm{E}+00$ \\
\hline 0.25 & $6.25000 \mathrm{E}-02$ & $6.25062 \mathrm{E}-02$ & $6.17924 \mathrm{E}-06$ & $6.25004 \mathrm{E}-02$ & $4.31777 \mathrm{E}-07$ & $6.25000 \mathrm{E}-02$ & $3.85920 \mathrm{E}-08$ \\
\hline 0.5 & $2.50000 \mathrm{E}-01$ & $2.50009 \mathrm{E}-01$ & $8.63030 \mathrm{E}-06$ & $2.50001 \mathrm{E}-01$ & $5.85276 \mathrm{E}-07$ & $2.50000 \mathrm{E}-01$ & $4.82884 \mathrm{E}-08$ \\
\hline 0.75 & $5.62500 \mathrm{E}-01$ & $5.62586 \mathrm{E}-01$ & $8.59383 \mathrm{E}-05$ & $5.62501 \mathrm{E}-01$ & $8.48308 \mathrm{E}-07$ & $5.62500 \mathrm{E}-01$ & $6.66913 \mathrm{E}-08$ \\
\hline 1 & $1.00000 \mathrm{E}+00$ & $1.00002 \mathrm{E}+00$ & $1.86691 \mathrm{E}-05$ & $1.00000 \mathrm{E}+00$ & $1.29128 \mathrm{E}-06$ & $1.00000 \mathrm{E}+00$ & $1.12686 \mathrm{E}-07$ \\
\hline
\end{tabular}


Numerical Solution of the Nonlocal Hammerstein-Volterra Integral Equation with Continuous...

Case (III) When $H(t, \varphi(t))=\varphi^{2}(t)$ : here we solve numerically (4.1) for different value of $(h=0.25,0.125,0.625)$ and $, \gamma(s, \varphi(s))=s \varphi^{2}(s), g(s, \varphi(s))=\varphi^{2}(s)$.

Table(11)

\begin{tabular}{|c|c|c|c|c|c|c|c|}
\hline \multicolumn{2}{|c|}{ CaseIII.1 : Trapezoidal method $H(t, \varphi(t))=\varphi^{2}(t), \gamma(s, \varphi(s))=s \varphi^{2}(s), g(s, \varphi(s))=\varphi^{2}(s)$} \\
\hline \multirow{2}{*}{$t$} & $\varphi$ & \multicolumn{2}{|c|}{$h=0.25, N=4$} & \multicolumn{2}{c|}{$h=0.125, N=8$} & \multicolumn{2}{c|}{$h=0.625, N=16$} \\
\cline { 3 - 8 } & & $\varphi^{T r}$ & $E^{T r}$ & $\varphi^{T r}$ & $E^{T r}$ & $\varphi^{T r}$ & $E^{T r}$ \\
\hline 0 & $0.00000 \mathrm{E}+00$ & $0.00000 \mathrm{E}+00$ & $0.00000 \mathrm{E}+00$ & $0.00000 \mathrm{E}+00$ & $0.00000 \mathrm{E}+00$ & $0.00000 \mathrm{E}+00$ & $0.00000 \mathrm{E}+00$ \\
\hline 0.25 & $6.25000 \mathrm{E}-02$ & $6.32013 \mathrm{E}-02$ & $7.01300 \mathrm{E}-04$ & $6.26802 \mathrm{E}-02$ & $1.80200 \mathrm{E}-04$ & $6.25454 \mathrm{E}-02$ & $4.54000 \mathrm{E}-05$ \\
\hline 0.5 & $2.50000 \mathrm{E}-01$ & $2.50369 \mathrm{E}-01$ & $3.69000 \mathrm{E}-04$ & $2.50095 \mathrm{E}-01$ & $9.50000 \mathrm{E}-05$ & $2.50024 \mathrm{E}-01$ & $2.40000 \mathrm{E}-05$ \\
\hline 0.75 & $5.62500 \mathrm{E}-01$ & $5.62790 \mathrm{E}-01$ & $2.90000 \mathrm{E}-04$ & $5.62574 \mathrm{E}-01$ & $7.40000 \mathrm{E}-05$ & $5.62519 \mathrm{E}-01$ & $1.90000 \mathrm{E}-05$ \\
\hline 1 & $1.00000 \mathrm{E}+00$ & $1.00031 \mathrm{E}+00$ & $3.10000 \mathrm{E}-04$ & $1.00008 \mathrm{E}+00$ & $8.00000 \mathrm{E}-05$ & $1.00002 \mathrm{E}+00$ & $2.00000 \mathrm{E}-05$ \\
\hline
\end{tabular}

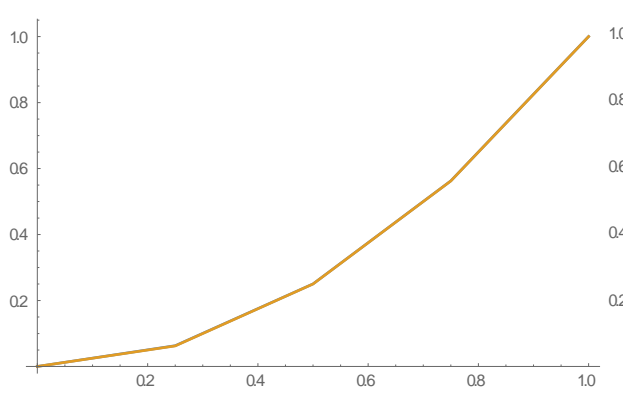

Fig.(11-i) $h=0.25, N=4$

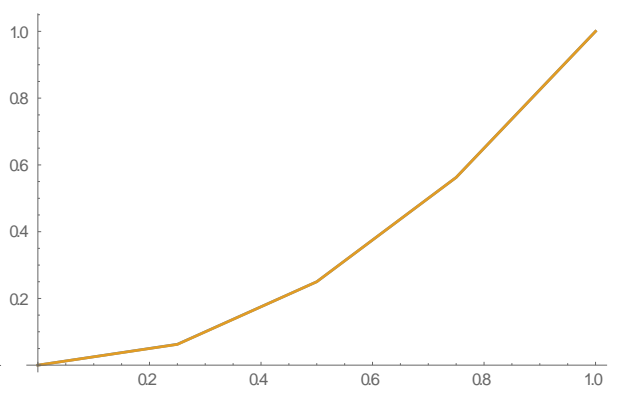

Fig. (11-ii) $h=0.125, N=8$

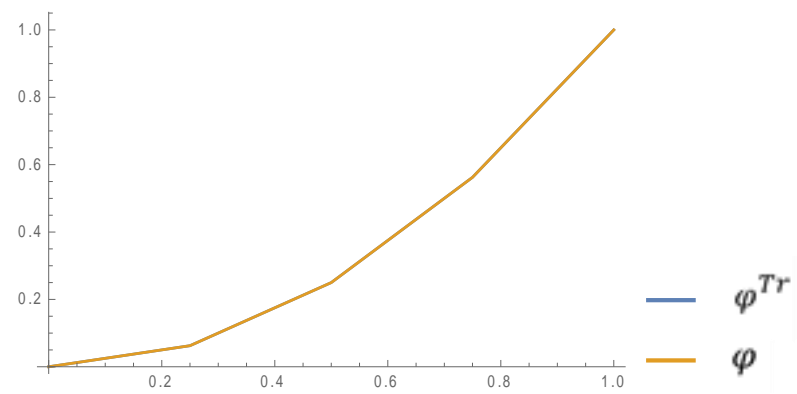

Fig.(11-iii) $h=0.625, N=16$

- Figs. (11) describe the relation between the exact solution and numerical solution, when $H(t, \varphi(t))=\varphi^{2}(t), \gamma(s, \varphi(s))=s \varphi^{2}(s), g(s, \varphi(s))=\varphi^{2}(s)$, using Trapezoidal method, with $\lambda=$ $0.01, \mu=0.001$ at $h=0.25, N=4 ; h=0.125, N=8 ; h=0.625, N=16$ in Fig. (11.i), Fig (11.ii) and Fig. (11.iii), respectively.

Table(12)

\begin{tabular}{|l|l|l|l|c|c|c|c|}
\hline \multicolumn{2}{|c|}{ Case III.1 : Simpson's method $H(t, \varphi(t))=\varphi^{2}(t), \gamma(s, \varphi(s))=s \varphi^{2}(s), g(s, \varphi(s))=\varphi^{2}(s)$} \\
\hline \multirow{2}{*}{$t$} & $\varphi$ & \multicolumn{2}{c|}{$h=0.25, N=4$} & \multicolumn{2}{c|}{$h=0.125, N=8$} & \multicolumn{2}{c|}{$h=0.625, N=16$} \\
\cline { 3 - 8 } & & $\varphi^{S}$ & $E^{S}$ & $\varphi^{S}$ & $E^{S}$ & $\varphi^{S}$ & $E^{S}$ \\
\hline 0 & $0.00000 \mathrm{E}+00$ & $0.00000 \mathrm{E}+00$ & $0.00000 \mathrm{E}+00$ & $0.00000 \mathrm{E}+00$ & $0.00000 \mathrm{E}+00$ & $0.00000 \mathrm{E}+00$ & $0.00000 \mathrm{E}+00$ \\
\hline 0.25 & $6.25000 \mathrm{E}-02$ & $6.25843 \mathrm{E}-02$ & $8.42710 \mathrm{E}-05$ & $6.25057 \mathrm{E}-02$ & $5.71716 \mathrm{E}-06$ & $6.25004 \mathrm{E}-02$ & $3.81175 \mathrm{E}-07$ \\
\hline 0.5 & $2.50000 \mathrm{E}-01$ & $2.50045 \mathrm{E}-01$ & $4.48635 \mathrm{E}-05$ & $2.50003 \mathrm{E}-01$ & $3.02943 \mathrm{E}-06$ & $2.50000 \mathrm{E}-01$ & $2.01423 \mathrm{E}-07$ \\
\hline 0.75 & $5.62500 \mathrm{E}-01$ & $5.62582 \mathrm{E}-01$ & $8.15022 \mathrm{E}-05$ & $5.62502 \mathrm{E}-01$ & $2.19748 \mathrm{E}-06$ & $5.62500 \mathrm{E}-01$ & $1.46384 \mathrm{E}-07$ \\
\hline 1 & $1.00000 \mathrm{E}+00$ & $1.00003 \mathrm{E}+00$ & $2.74895 \mathrm{E}-05$ & $1.00000 \mathrm{E}+00$ & $1.85389 \mathrm{E}-06$ & $1.00000 \mathrm{E}+00$ & $1.27695 \mathrm{E}-07$ \\
\hline
\end{tabular}



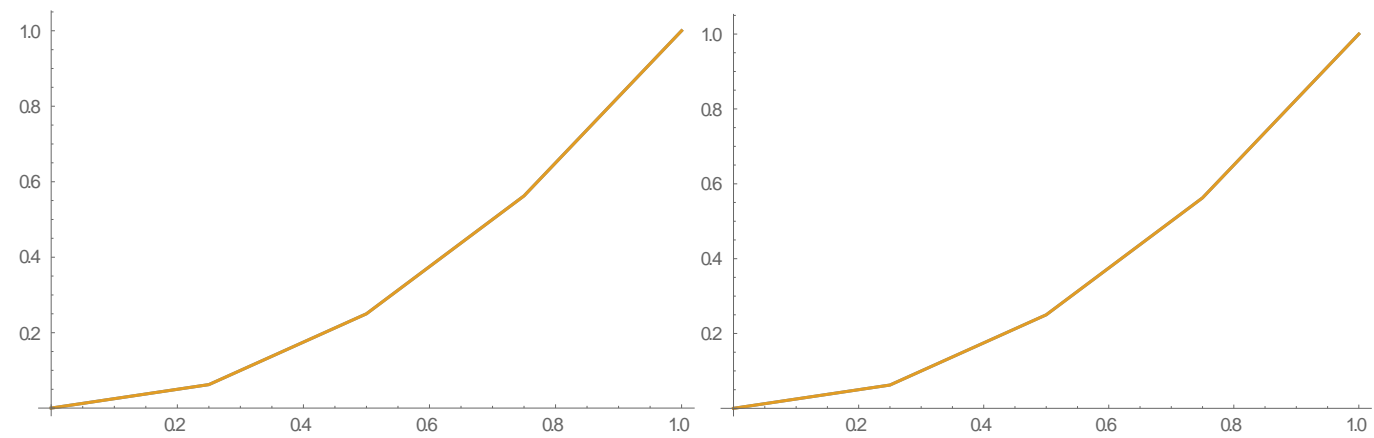

Fig.(12-i) $h=0.25, N=4$ Fig.(12-ii) $h=0.125, N=8$

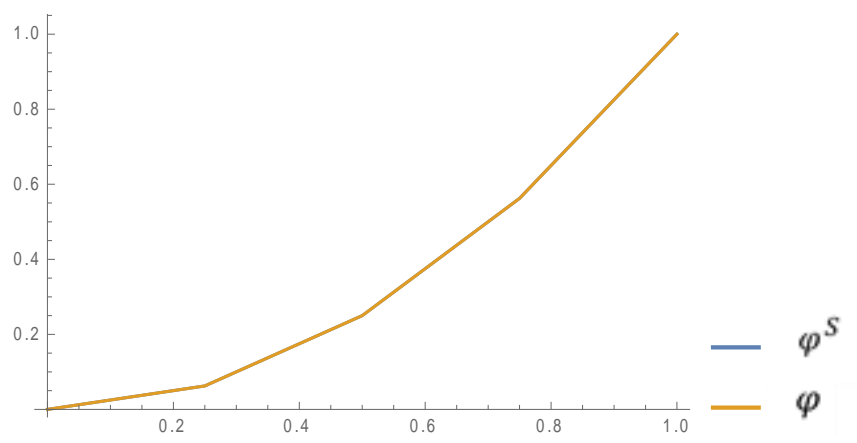

Fig.(12-iii) $h=0.625, N=16$

- Figs. (12) describe the relation between the exact solution and numerical solution, when $H(t, \varphi(t))=$ $\varphi^{2}(t), \gamma(s, \varphi(s))=s \varphi^{2}(s), g(s, \varphi(s))=\varphi^{2}(s)$, using Simpson's method with $\lambda=0.01, \mu=0.001$ at $h=0.25, N=4 \quad ; \quad h=0.125, N=8 \quad ; h=0.625, N=16$ in Fig. (13.i), Fig (13.ii) and Fig. (13.iii),respectively.

(III.2) When $H(t, \varphi(t))=\varphi^{2}(t)$ and, $\gamma(s, \varphi(s))=s \varphi^{2}(s)$ and, $g(s, \varphi(s))=\varphi(s)$.

Table(13)

\begin{tabular}{|l|c|c|c|c|c|c|c|}
\hline \multicolumn{2}{|c|}{ Case III.2: Trapezoidal method $H(t, \varphi(t))=\varphi^{2}(t), \gamma(s, \varphi(s))=s \varphi^{2}(s), g(s, \varphi(s))=\varphi(s)$} \\
\hline \multirow{2}{*}{$t$} & $\varphi$ & \multicolumn{2}{c|}{$h=0.25, N=4$} & \multicolumn{2}{c|}{$h=0.125, N=8$} & \multicolumn{2}{c|}{$h=0.625, N=16$} \\
\cline { 3 - 8 } & & $\varphi^{T r}$ & $E^{T r}$ & $\varphi^{T r}$ & $E^{T r}$ & $\varphi^{T r}$ & $E^{T r}$ \\
\hline 0 & $0.00000 \mathrm{E}+00$ & $0.00000 \mathrm{E}+00$ & $0.00000 \mathrm{E}+00$ & $0.00000 \mathrm{E}+00$ & $0.00000 \mathrm{E}+00$ & $0.00000 \mathrm{E}+00$ & $0.00000 \mathrm{E}+00$ \\
\hline 0.25 & $6.25000 \mathrm{E}-02$ & $6.32190 \mathrm{E}-02$ & $7.19000 \mathrm{E}-04$ & $6.26847 \mathrm{E}-02$ & $1.84700 \mathrm{E}-04$ & $6.25465 \mathrm{E}-02$ & $4.65000 \mathrm{E}-05$ \\
\hline 0.5 & $2.50000 \mathrm{E}-01$ & $2.50393 \mathrm{E}-01$ & $3.93000 \mathrm{E}-04$ & $2.50100 \mathrm{E}-01$ & $1.00000 \mathrm{E}-04$ & $2.50025 \mathrm{E}-01$ & $2.50000 \mathrm{E}-05$ \\
\hline 0.75 & $5.62500 \mathrm{E}-01$ & $5.62795 \mathrm{E}-01$ & $2.95000 \mathrm{E}-04$ & $5.62575 \mathrm{E}-01$ & $7.50000 \mathrm{E}-05$ & $5.62519 \mathrm{E}-01$ & $1.90000 \mathrm{E}-05$ \\
\hline 1 & $1.00000 \mathrm{E}+00$ & $1.00026 \mathrm{E}+00$ & $2.60000 \mathrm{E}-04$ & $1.00007 \mathrm{E}+00$ & $7.00000 \mathrm{E}-05$ & $1.00002 \mathrm{E}+00$ & $2.00000 \mathrm{E}-05$ \\
\hline
\end{tabular}

Table(14)

\begin{tabular}{|c|c|c|c|c|c|c|c|}
\hline \multicolumn{2}{|c|}{ Case III.2: Simpson's method $H(t, \varphi(t))=\varphi^{2}(t), \gamma(s, \varphi(s))=s \varphi^{2}(s), g(s, \varphi(s))=\varphi(s)$} \\
\hline \multirow{2}{*}{$t$} & $\varphi$ & \multicolumn{2}{c|}{$h=0.25, N=4$} & \multicolumn{2}{c|}{$h=0.125, N=8$} & \multicolumn{2}{c|}{$h=0.625, N=16$} \\
\cline { 3 - 8 } & & $\varphi^{S}$ & $E^{S}$ & $\varphi^{S}$ & $E^{S}$ & $\varphi^{S}$ & $E^{S}$ \\
\hline 0 & $0.00000 \mathrm{E}+00$ & $0.00000 \mathrm{E}+00$ & $0.00000 \mathrm{E}+00$ & $0.00000 \mathrm{E}+00$ & $0.00000 \mathrm{E}+00$ & $0.00000 \mathrm{E}+00$ & $0.00000 \mathrm{E}+00$ \\
\hline 0.25 & $6.25000 \mathrm{E}-02$ & $6.26021 \mathrm{E}-02$ & $1.02057 \mathrm{E}-04$ & $6.25056 \mathrm{E}-02$ & $5.60898 \mathrm{E}-06$ & $6.25004 \mathrm{E}-02$ & $3.70656 \mathrm{E}-07$ \\
\hline 0.5 & $2.50000 \mathrm{E}-01$ & $2.50042 \mathrm{E}-01$ & $4.16962 \mathrm{E}-05$ & $2.50003 \mathrm{E}-01$ & $2.82946 \mathrm{E}-06$ & $2.50000 \mathrm{E}-01$ & $1.87841 \mathrm{E}-07$ \\
\hline 0.75 & $5.62500 \mathrm{E}-01$ & $5.62586 \mathrm{E}-01$ & $8.64287 \mathrm{E}-05$ & $5.62502 \mathrm{E}-01$ & $1.89923 \mathrm{E}-06$ & $5.62500 \mathrm{E}-01$ & $1.27659 \mathrm{E}-07$ \\
\hline 1 & $1.00000 \mathrm{E}+00$ & $1.00002 \mathrm{E}+00$ & $2.10063 \mathrm{E}-05$ & $1.00000 \mathrm{E}+00$ & $1.43921 \mathrm{E}-06$ & $1.00000 \mathrm{E}+00$ & $9.90302 \mathrm{E}-08$ \\
\hline
\end{tabular}

\section{Conclusions}

From the above results and others results we have obtained, we can see that the proposed methods are efficient and accurate, also we note the following:

1. The value of absolute error is decreasing when the value of $h$ decreases in the two methodsfor all cases of studies.

2 The smallest error is obtained, with respect to the two methodsfor all cases of studies, when the nonlocal function in the nonlinear formwhen $\mu \leq 0.001$. 
3. The error of theSimpson's method is smaller than the corresponding error of the Trapezoidal method for all cases of studies. So,Simpson's method is the best.

4. The absolute value of the error when the memory term $H(t, \phi(t))$ takes a nonlinear form is less than the corresponding error of the linear form in the two methods forseveral forms of $\gamma(t, \varphi(t))$ and $g(t, \varphi(t))$.

5. When the memory term $H(t, \varphi(t))=0$ the absolute value of the error is large when $\mu \leq 0.001(\mu \ll 1)$ for several forms of $\gamma(t, \varphi(t))$ and $g(t, \varphi(t))$.

6. The value of absolute error is decreasing when the value of $\mu$ increases when the memory $\operatorname{term} H(t, \varphi(t))=0$ in the two methods for several forms of $\gamma(t, \varphi(t))$ and $g(t, \varphi(t))$.

7. In the nonlocal integral equations $\mu$ is called the phase-lag of the integral equations.

8. . TheMax. E. and Min. E. in all casesof studiesare given asfollows:

(I). First: when the memory term vanishes.

(I.1). when $H(t, \varphi(t))=0, \gamma(s, \varphi(s))=s \varphi^{2}(s), g(s, \varphi(s))=\varphi^{2}(s)$

1. For the Trapezoidal method $H(t, \varphi(t))=0, \gamma(s, \varphi(s))=s \varphi^{2}(s), g(s, \varphi(s))=\varphi^{2}(s)$ we have Max. E. and Min. E. in Table (1) at $(h=0.625, \lambda=0.01)$ as follow:

when $\mu=0.1$ : (at $\mathrm{t}=1$ ) 4.10000E-04and (at $\mathrm{t}=0$ ) $0.00000 \mathrm{E}+00$, respectively.

when $\mu=0.5$ : (at $\mathrm{t}=1) 8.00000 \mathrm{E}-05$ and (at $\mathrm{t}=0) 0.00000 \mathrm{E}+00$, respectively.

when $\mu=1:($ at $\mathrm{t}=1$ ) 4.00000E-05and (at $\mathrm{t}=0$ ) $0.00000 \mathrm{E}+00$, respectively.

2 For the Simpson'smethod $H(t, \varphi(t))=0, \gamma(s, \varphi(s))=s \varphi^{2}(s), g(s, \varphi(s))=\varphi^{2}(s)$ we have

Max. E. and Min. E.in Table (2) at $(h=0.625, \lambda=0.01)$ as follow:

when $\mu=0.1$ :(at $\mathrm{t}=1$ ) 6.74107E-06and (at $\mathrm{t}=0$ ) $0.00000 \mathrm{E}+00$, respectively.

when $\mu=0.5$ : (at $\mathrm{t}=1$ ) 6.27828E-07and (at $\mathrm{t}=0$ ) $0.00000 \mathrm{E}+00$, respectively.

when $=1:($ at $\mathrm{t}=1) 2.70709 \mathrm{E}-07$ and $($ at $\mathrm{t}=0) 0.00000 \mathrm{E}+00$, respectively.

(I.2). when $H(t, \varphi(t))=0, \gamma(s, \varphi(s))=s \varphi^{2}(s), g(s, \varphi(s))=\varphi(s)$

1- For the Trapezoidal method $H(t, \varphi(t))=0, \gamma(s, \varphi(s))=s \varphi^{2}(s), g(s, \varphi(s))=\varphi(s)$ we have Max. E and Min. E.in Table (3) at $(h=0.625, \lambda=0.01)$ as follow:

when $\mu=0.1$ : (at $\mathrm{t}=1$ ) 3.40000E-04and (at $\mathrm{t}=0$ ) $0.00000 \mathrm{E}+00$, respectively.

when $\mu=0.5$ : (at $\mathrm{t}=1$ ) 7.00000E-05and (at $\mathrm{t}=0$ ) $0.00000 \mathrm{E}+00$, respectively.

when $=1:($ at $\mathrm{t}=1$ ) 3.00000E-05and (at $\mathrm{t}=0) 0.00000 \mathrm{E}+00$, respectively.

2- For theSimpson'smethod $H(t, \varphi(t))=0, \gamma(s, \varphi(s))=s \varphi^{2}(s), g(s, \varphi(s))=\varphi(s)$ we haveMax. E. and Min. E.in Table (4) at $(h=0.625, \lambda=0.01)$ as follow:

when $\mu=0.1$ : (at $\mathrm{t}=1$ ) $1.87988 \mathrm{E}-07$ and (at $\mathrm{t}=0$ ) $0.00000 \mathrm{E}+00$, respectively. when $\mu=0.5$ : (at $\mathrm{t}=1$ ) 1.31819E-07and (at $\mathrm{t}=0$ ) $0.00000 \mathrm{E}+00$, respectively. when $=1:($ at $\mathrm{t}=1) 9.60355 \mathrm{E}-08$ and $($ at $\mathrm{t}=0) 0.00000 \mathrm{E}+00$, respectively.

\section{(II).Second: when the memory term is linear}

(II.1) when $H(t, \varphi(t))=t \varphi(t), \gamma(s, \varphi(s))=s \varphi^{2}(s), g(s, \varphi(s))=\varphi^{2}(s)$

1- For the Trapezoidal method $H(t, \varphi(t))=t \varphi(t), \gamma(s, \varphi(s))=s \varphi^{2}(s), g(s, \varphi(s))=\varphi^{2}(s)$ we have Max. E. and Min. E.in Table (5) at $(\mu=0.001, \lambda=0.01)$ as follow:

when $h=0.25$ : (at $\mathrm{t}=1) 6.14772 \mathrm{E}-04$ and (at $\mathrm{t}=0) 0.00000 \mathrm{E}+00$, respectively. when $h=0.125$ : (at $\mathrm{t}=1) 1.56278 \mathrm{E}-04$ and (at $\mathrm{t}=0) 0.00000 \mathrm{E}+00$, respectively. when $h=0.625:($ at $\mathrm{t}=1) 3.92329 \mathrm{E}-05$ and $($ at $\mathrm{t}=0)$ 0.00000E+00, respectively

2- For the Simpson'smethod $H(t, \varphi(t))=t \varphi(t), \gamma(s, \varphi(s))=s \varphi^{2}(s), g(s, \varphi(s))=\varphi^{2}(s)$ we have Max. E. and Min. E.in Table (6) at $(\mu=0.001, \lambda=0.01)$ as follow:

when $h=0.25$ : (at $\mathrm{t}=1$ ) 5.52195E-05and (at $\mathrm{t}=0$ ) $0.00000 \mathrm{E}+00$, respectively.

when $h=0.125$ : (at $\mathrm{t}=1$ ) 3.78196E-06and (at $\mathrm{t}=0$ ) $0.00000 \mathrm{E}+00$, respectively.

when $h=0.625$ : $($ at $\mathrm{t}=1)$ 2.75956E-07and (at $\mathrm{t}=0) 0.00000 \mathrm{E}+00$, respectively

(II.2) when $H(t, \varphi(t))=t \varphi(t), \gamma(s, \varphi(s))=s \varphi^{2}(s), g(s, \varphi(s))=\varphi(s)$

1- For the Trapezoidal method $H(t, \varphi(t))=t \varphi(t), \gamma(s, \varphi(s))=s \varphi^{2}(s), g(s, \varphi(s))=\varphi(s)$ we have Max. E. and Min. E.in Table (7) at $(\mu=0.001, \lambda=0.01)$ as follow: 
when $h=0.25$ : (at $\mathrm{t}=1$ ) 5.13197E-04and (at $\mathrm{t}=0$ ) $0.00000 \mathrm{E}+00$, respectively. when $h=0.125$ : (at $\mathrm{t}=1) 1.30343 \mathrm{E}-04$ and (at $\mathrm{t}=0) 0.00000 \mathrm{E}+00$, respectively. when $h=0.625$ : (at $\mathrm{t}=1$ ) 3.27157E-05and (at $\mathrm{t}=0$ ) 0.00000E+00, respectively.

2- For the Simpson'smethod $H(t, \varphi(t))=t \varphi(t), \gamma(s, \varphi(s))=s \varphi^{2}(s), g(s, \varphi(s))=\varphi(s)$ we have Max. E. and Min. E.in Table (8) at $(\mu=0.001, \lambda=0.01)$ as follow:

when $h=0.25$ : (at $\mathrm{t}=0.75$ ) 1.29708E-04and (at $\mathrm{t}=0$ ) $0.00000 \mathrm{E}+00$, respectively. when $h=0.125$ : (at $\mathrm{t}=1$ ) 2.92484E-06and (at $\mathrm{t}=0) 0.00000 \mathrm{E}+00$, respectively. when $h=0.625$ : $($ at $\mathrm{t}=1) 2.10280 \mathrm{E}-07$ and $($ at $\mathrm{t}=0) 0.00000 \mathrm{E}+00$, respectively

(II.3) when $H(t, \varphi(t))=t \varphi(t), \gamma(s, \varphi(s))=\varphi(s), g(s, \varphi(s))=\varphi^{2}(s)$

1- For the Trapezoidal method $H(t, \varphi(t))=t \varphi(t), \gamma(s, \varphi(s))=\varphi(s), g(s, \varphi(s))=\varphi^{2}(s)$ we have Max. E. and Min. E.in Table (9) at $(\mu=0.001, \lambda=0.01)$ as follow:

when $h=0.25$ : (at $\mathrm{t}=1) 4.66820 \mathrm{E}-04$ and (at $\mathrm{t}=0) 0.00000 \mathrm{E}+00$, respectively. when $h=0.125$ : (at $\mathrm{t}=1) 1.17498 \mathrm{E}-04$ and (at $\mathrm{t}=0) 0.00000 \mathrm{E}+00$, respectively. when $h=0.625$ : (at $\mathrm{t}=1$ ) 2.94241E-05and (at $\mathrm{t}=0)$ 0.00000E+00, respectively.

2- For the Simpson'smethod $H(t, \varphi(t))=t \varphi(t), \gamma(s, \varphi(s))=\varphi(s), g(s, \varphi(s))=\varphi^{2}(s)$ we have Max. E. and Min. E.in Table (10) at $(\mu=0.001, \lambda=0.01)$ as follow:

when $h=0.25$ : (at $\mathrm{t}=0.75$ ) 8.59383E-05and (at $\mathrm{t}=0$ ) $0.00000 \mathrm{E}+00$, respectively. when $h=0.125:($ at $\mathrm{t}=1) 1.29128 \mathrm{E}-06$ and $($ at $\mathrm{t}=0) 0.00000 \mathrm{E}+00$, respectively. when $h=0.625:($ at $\mathrm{t}=1) 1.12686 \mathrm{E}-07$ and $($ at $\mathrm{t}=0) 0.00000 \mathrm{E}+00$, respectively.

(III).Third: when the memory term is nonlinear.

(III.1) when $H(t, \varphi(t))=\varphi^{2}(t), \gamma(s, \varphi(s))=s \varphi^{2}(s), g(s, \varphi(s))=\varphi^{2}(s)$

1- For the Trapezoidal method $H(t, \varphi(t))=\varphi^{2}(t), \gamma(s, \varphi(s))=s \varphi^{2}(s), g(s, \varphi(s))=\varphi^{2}(s)$ we have Max. E. and Min. E.in Table (11) at $(\mu=0.001, \lambda=0.01)$ as follow:

when $h=0.25$ : (at $\mathrm{t}=0.25$ ) 7.01300E-04and (at $\mathrm{t}=0$ ) $0.00000 \mathrm{E}+00$, respectively. when $h=0.125:($ at $\mathrm{t}=0.25) 1.80200 \mathrm{E}-04$ and $($ at $\mathrm{t}=0) 0.00000 \mathrm{E}+00$, respectively. when $h=0.625:($ at $\mathrm{t}=0.25) 4.54000 \mathrm{E}-05$ and (at $\mathrm{t}=0) 0.00000 \mathrm{E}+00$, respectively.

2- For the Simpson'smethod $H(t, \varphi(t))=\varphi^{2}(t), \gamma(s, \varphi(s))=s \varphi^{2}(s), g(s, \varphi(s))=\varphi^{2}(s)$ we have Max. E. and Min. E.in Table (12) at $(\mu=0.001, \lambda=0.01)$ as follow:

when $h=0.25$ : (at $\mathrm{t}=0.25$ ) 8.42710E-05and (at $\mathrm{t}=0) 0.00000 \mathrm{E}+00$, respectively. when $h=0.125$ : (at $\mathrm{t}=0.25)$ 5.71716E-06and (at $\mathrm{t}=0) 0.00000 \mathrm{E}+00$, respectively. when $h=0.625$ : (at $\mathrm{t}=0.25$ ) 3.81175E-07and (at $\mathrm{t}=0$ ) 0.00000E+00, respectively.

(III.2) when $H(t, \varphi(t))=\varphi^{2}(t), \gamma(s, \varphi(s))=s \varphi^{2}(s), g(s, \varphi(s))=\varphi(s)$

1- For the Trapezoidal method $H(t, \varphi(t))=\varphi^{2}(t), \gamma(s, \varphi(s))=s \varphi^{2}(s), g(s, \varphi(s))=\varphi(s)$ we have Max. E. and Min. E.in Table (13) at $(\mu=0.001, \lambda=0.01)$ as follow:

when $h=0.25$ : (at $\mathrm{t}=0.25$ ) 7.19000E-04and (at $\mathrm{t}=0$ ) $0.00000 \mathrm{E}+00$, respectively.

when $h=0.125:($ at $\mathrm{t}=0.25) 1.84700 \mathrm{E}-04$ and $($ at $\mathrm{t}=0) 0.00000 \mathrm{E}+00$, respectively.

when $h=0.625:($ at $\mathrm{t}=0.25$ ) 4.65000E-05and (at $\mathrm{t}=0) 0.00000 \mathrm{E}+00$, respectively.

2- For the Simpson's method $H(t, \varphi(t))=\varphi^{2}(t), \gamma(s, \varphi(s))=s \varphi^{2}(s), g(s, \varphi(s))=\varphi(s)$, we have Max. E. and Min. E.in Table (14) at $(\mu=0.001, \lambda=0.01)$ as follow:

when $h=0.25$ : (at $\mathrm{t}=0.25$ ) 1.02057E-04and (at $\mathrm{t}=0$ ) 0.00000E+00, respectively.

when $h=0.125:($ at $\mathrm{t}=0.25) 5.60898 \mathrm{E}-06$ and $($ at $\mathrm{t}=0) 0.00000 \mathrm{E}+00$, respectively.

when $h=0.625$ :(at $\mathrm{t}=0.25$ ) 3.70656E-07and (at $\mathrm{t}=0) 0.00000 \mathrm{E}+00$, respectively.

\section{References}

[1] A. Shidfar et al, A series solution of the nonlinear Volterra and Fredholmintegrodifferentialequations, Commun Nonlinear SciNumerSimulat 15 (2010) 205-215.

[2] A. Aky uz, M. Sezer, A Taylor polymial approach for solving high-order linear Fredholm integro differential equations in the most general form, International Journal of Computer Mathematics (2007)527-539.

[3] N. Lungu, I. A. Rus, On a functional Volterra- Fredholm integral equation viaPicard operator, Journal of Mathematical Inequalities, 3, (4) (2009), 519-527.

[4] M. A. Abdou, M. M. EL-Borai, M. M. El-Kojok, Toeplitz matrix method and nonlinear integral equation of Hammerstein type, J. Comp. Appl. Math.Vol. 223, (2009) 765 - 776. 
[5] M. A. Abdou, A. A. Badr, M. M. El-Kojok, On the solution of mixed nonlinear integral equation, Appl. Math. Compute. 217(2011) 5466-5475.

[6] H. Wang, H. M. Fu, H.F. Zhang and et al, Numerical approach to wind ripple in desert, International Journal of Nonlinear Science and Numerical Simulation 8(2007)223-228.

[7] R. Kangro, P. Oja, Convergence of spline collection for Volterra integral equation, Appl. Num. Math. 58(2008)1434-1447.

[8] T. Diogo, P. Lima, Super convergence of collocation methods for class of weakly singular integral equations, J. Cam. Appl. Math. 218(2008) 307-316.

[9] M. A. Abdou, Fredholm - Volterra equation of the first kind and contact problem, Appl. Math. Compute. 125 (2002) 177 -193.

[10] M. A. Abdou, Fredholm integral equation with potential kernel and its structure resolvent, Appl. Math. Compute. 107 (2000) 169180.

[11] J. P. Kauthen, Continuous time collection for Volterra-Fredholm integral equations, Numerische Math. 56 (1989) 409-424.

[12] M. A. Abdou, Integral equation of mixed type and integrals of orthogonal polynomials, J. Comp. Appl. Math. 138 (2002) 273 285 .

[13] N.I. Muskhelishvili, Singular integral equations, Noordhoff (1953).

[14] G. Ya. Popov, Contact problems for a linearly deformable base, Kiev, Odessa (1982).

[15] F.G.Tricomi, Integral equations, N.Y. (1985)

[16] H. Hochstadt, Integral equations, N.Y. London (1971).

[17] C. D. Green, Integral equation methods, N.Y. (1969).

[18] K.E. Atkinson, A Survey of Numerical Method for the Solution of Fredholm Integral Equation of the Second Kind, SIAM, Philadelphia, 2012.

[19] L.M. Delves and J.L. Mohamed, Computational Methods for Integral Equations,Philadelphia, 2011

[20] M. A. Golberg, Numerical Solution of Integral Equation, Plenum, N. Y. 1990.

[21] P. Linz, Analytical and Numerical methods for Volterra equations, SIAM, Philadelphia, 1985. 